\title{
The Plancherel Theorem for Biinvariant Hilbert Spaces
}

By

\author{
Bernhard Krötz*
}

\section{Introduction}

Let $\mathrm{g}$ be a finite dimensional Lie algebra over the real numbers. An element $\mathrm{X} \in \mathrm{g}$ is called elliptic if ad $X$ operates semisimply with purely imaginary spectrum. We assume that $\mathfrak{g}$ admits an open Inn $(\mathfrak{g})$-invariant convex cone $W$ with non-empty elliptic interior $W^{0}$. This assumption is satisfied whenever $\mathrm{g}$ is hermitian or compact non- semisimple, but also for a large class of nonreductive Lie algebras, for instance the Jacobi algebras $\mathfrak{h}_{n} \rtimes \mathfrak{g p}(n, \mathbb{R}), n \in \mathbb{N}$, where $\mathfrak{h}_{n}$ denotes the $2 n+1$ dimensional Heisenberg algebra.

If $G$ is a connected Lie group with Lie algebra $\mathfrak{g}$, we build the complex Ol'shanskiı semigroup $\Gamma_{G}(W)=G \operatorname{Exp}(i W)$, which may be understood as a quotient by $\pi_{1}(G)$ of the universal covering semigroup of $\left\langle\exp _{G \mathbb{C}}(g+i W)\right\rangle$, where $G \mathbb{C}$ denotes a simply connected complex group with Lie algebra $\mathfrak{g}_{\mathbb{C}}$. A non-empty connected $G \times G$-invariant open subset $D \subseteq \Gamma_{\mathrm{G}}\left(W^{0}\right)$ is called a biinvariant domain. Then $D=G \operatorname{Exp}\left(D_{h}\right)$, where $D_{h} \subseteq i W^{0}$ is a connected open subset. We note that $D$ is a complex submanifold of $\Gamma_{\mathrm{G}}\left(W^{0}\right)$ and write $\operatorname{Hol}(D)$ for the space of holomorphic functions on $D$ equipped with the topology of compact convergence.

A biinvariant Hilbert space is a Hilbert space $\mathscr{H}$ sitting continuously in Hol $(D)$ on which $G \times G$ acts unitarily via

$$
\left((\lambda \otimes \rho)\left(g_{1}, g_{2}\right) . f\right)(z)=f\left(g_{1}^{-1} z g_{2}\right)
$$

for $g_{1}, g_{2} \in G, f \in \mathscr{H}, z \in D$. As the inclusion mapping $\mathscr{H} \hookrightarrow \operatorname{Hol}(D)$ is continuous, all point evaluations $K_{z}: \mathscr{H} \rightarrow \mathbb{C}, f \mapsto f(z)$ are continuous.

Communicated by M. Kashiwara, May18, 1998.

1991 Math. Subject Classifications: 22E45

* Math. Inst., TU Clausthal, Erzstr, 1, 38678 Clausthal-Zellerleld, Germany

mabk@math.tu-clausthal.de 
Therefore $\mathscr{H}$ is a reproducing kernel Hilbert space with kernel $K(z, w)=$ $\left\langle K_{w}, K_{z}\right\rangle$, also denoted by $\mathscr{H}_{K}$. It is easy to see that $K$ is biinvariant, i.e., $K\left(g_{1} z g_{2}\right.$, $w)=K\left(z, g_{1}^{-1} w g_{2}^{-1}\right)$ holds for all $g_{1}, g_{2} \in G, z, w \in D$, and that the prescription $K$ $\mapsto \mathscr{H}_{K}$ defines a correspondence between holomorphic biinvariant positive definite

kernels and biinvariant Hilbert spaces.

Now the natural question arises how $\left(\lambda \otimes \rho, \mathscr{H}_{K}\right)$ decomposes into irreducible representations, i.e., we ask for a Plancherel Theorem for Biinvariant Hilbert Spaces. Using the considerations from above one can show that this problem is more or less equivalent to the problem of decomposing a biinvariant kernel $K$ into its extremal constituents.

One may consider a complex Ol'shanskir semigroup as a complexification of the symmetric space $(G \times G) / \Delta(G) \cong G$, where $\Delta(G)=\{(g, g) \in G \times G: g \in G\}$ is the diagonal, and every biinvariant domain as a $(G \times G)$-invariant domain of this complexification. Thus biinvariant Hilbert spaces are a special class of invariant Hilbert spaces of holomorphic functions on certain complexifications of symmetric spaces (cf. [KNÓ97] for more details). Such invariant Hilbert spaces have already been studied in [HÓø91], where a Plancherel Formula for the Hardy space corresponding to an affine symmetric space is proved. But it seems to be too early for a discussion of invariant Hilbert spaces in general, because a detailed knowledge of spherical highest weight representations and their associated characters is needed; a theory which is still in development (see [KrNe96], [KNÓ97.98], [Kr98b, d] for the latest results).

The most powerful method in dealing with invariant Hilbert spaces is to embed the invariant Hilbert space into a biinvariant one and then using the results of the biinvariant setting. Thus it is inevitable to understand biinvariant Hilbert spaces first. Since we kept large parts of this paper general, it provides many tools to deal with invariant Hilbert spaces as soon as the theory of spherical highest weight representations is sufficiently far developed.

Plancherel Theorems have already been proved for special classes of biinvariant Hilbert spaces, namely for the Hardy spaces corresponding to linear hermitian (cf. [O182], [O191], [O195]) and solvable Lie groups (cf. [HiÖ192]) as well as for a Bergman space associated to $\operatorname{Sp}(n, \mathbb{R})$ (cf. [Pe96]). The Plancherel Theorem for Hardy spaces for arbitrary $G$ was suggested in [Ne95]. But in all cases established so far one has needed very special assumptions on the group, for example compactness of the center if the group is reductive to guarantee discrete decomposability. This is because in this special case the "abstract" desintegration theory of $C^{*}$-algebras (cf. [Ne94], [Kr98c]) really gives "concrete" realizations. But in general the $C^{*}$-theory fails for giving explicit answers. In this paper we give a sufficiently concrete description of the Plancherel theorem for all applications. It will serve as a foundation for a general treatment of Hardy and Bergman spaces (cf. [Kr98a]). 
The paper is organised as follows:

I. Conuclear spaces and integral representations

II. Positive definite kernels on complex manifolds

III. Biinvariant domains in complex Ol'shanskī semigroups

IV. The Plancherel Theorem for Biinvariant Hilbert Spaces.

One main ingredient in the proof of the Plancherel Theorem is Thomas' Integral Representation Theorem for Conuclear Cones (cf. [Th94]). Section I is devoted to a brief discussion of conuclear spaces and explains the Integral Representation Theorem.

Let $M$ be a separable complex manifold and $\bar{M}$ the same manifold equipped with the opposite complex structure. Let $S$ be an involutive semigroup acting on $M$ by holomorphic mappings from the left, $V$ a finite dimensional Hilbert space, $B(V)$ the space of linear operators on $V$ and $\mathscr{P}\left(M^{2}, V\right)$ the convex cone of holomorphic positive definite $B(V)$-valued kernels. For each $K \in \mathscr{P}\left(M^{2}, V\right)$ we write $\mathscr{H}_{K} \subseteq \operatorname{Hol}(M, V)$ for the corresponding reproducing kernel Hilbert space of holomorphic functions. A kernel $K \in \mathscr{P}\left(M^{2}, V\right)$ is called $S$-invariant if $K(s . z, w)$ $=K\left(z, s^{*} . w\right)$ holds for all $s \in S, z, w \in M$, and we denote by $\mathscr{P}\left(M^{2}, V\right)_{i}$ the convex subcone of $\mathscr{P}\left(M^{2}, V\right)$ of all $S$-invariant elements. We call $K \in \mathscr{P}\left(M^{2}, V\right)_{i}$ exponentially bounded if for each $s \in S$ there exists a positive number $\alpha_{K}(s)$ such that

$$
\langle K(s . z, s . z) . v, v\rangle \leq \alpha_{K}(s)\langle K(z, z) . v, v\rangle
$$

holds for all $z \in M$ and $v \in V$. In this case the prescription

$$
\pi_{K}: S \rightarrow B\left(\mathscr{H}_{K}\right),\left(\pi_{K}(s) . f\right)(z)=f\left(s^{*} . z\right)
$$

gives rise to a representation of $S$.

In Section II we show that the Integral Representation Theorem applies to the closed convex subcones of $\mathscr{P}\left(M^{2}, V\right)_{i}$ in the conuclear space Hol $(M \times \bar{M}, V)$. It turns out that each exponentially bounded kernel $K \in \mathscr{P}\left(M^{2}, V\right)_{i}$ with $\left(\pi_{K}\right.$, $\mathscr{H}_{K}$ ) multiplicity-free has an integral representation

$$
K=\int_{\operatorname{Ext}\left(\mathscr{P}\left(M^{2}, V\right)_{t}\right)} Q d \mu(Q),
$$

where $\mu$ is a uniquely determined Radon measure supported on a suitable section on the subcone of extreme generators $\operatorname{Ext}\left(\mathscr{P}\left(M^{2}, V\right)_{i}\right)$ of $\mathscr{P}\left(M^{2}, V\right)_{i}$.

Moreover we show that there is a unitary equivalence of $S$-modules

$$
\left(\pi_{K}, \mathscr{H}_{K}\right) \rightarrow\left(\int_{\operatorname{Ext}\left(\mathscr{P}\left(M^{2}, V\right)_{\imath}\right)}^{\oplus} \pi_{Q} d \mu(Q), \int_{\operatorname{Ext}\left(\mathscr{P}\left(M^{2}, V\right)_{\imath}\right)}^{\oplus} \mathscr{H}_{Q} d \mu(Q)\right) .
$$

Section III summarizes the main facts concerning the convex and complex geometry of biinvariant domains mainly due to K.-H. Neeb (cf. [Ne98], [Ne99]) .

In Section IV we finally prove the Plancherel Theorem for Biinvariant 
Hilbert Spaces (cf. Theorem IV.12). It says that each $K \in \mathscr{P}\left(D^{2}\right)_{i}$ can uniquely be written as

$$
K=\int_{H W\left(G, \Delta^{+}\right)} K^{\lambda} d \mu(\lambda)
$$

Here $\mu$ denotes a Radon measure on the Borel subset of highest weights $H W(G$, $\left.\Delta^{+}\right) \subseteq i \mathrm{t}^{*}$ of $G$, where $\mathrm{t}$ denotes a compactly embedded Cartan subalgebra of $\mathrm{g}$ and $\Delta^{+}$a certain positive system determined by $D$. Further $K^{\lambda} \in \operatorname{Ext}\left(\mathscr{P}\left(D^{2}\right)_{i}\right)$ is defined by $K^{\lambda}(s, t)=\Theta_{\lambda}\left(s t^{*}\right)$ for $s, t \in D$, where $\Theta_{\lambda}$ is the character of the unitarizable highest weight module $L(\lambda)$ considered as a holomorphic function on $\Gamma_{G}\left(W^{0}\right)$. On the level of $G \times G$-modules we have a unitary equivalence

$$
\left(\lambda \otimes \rho, \mathscr{H}_{K}\right) \rightarrow\left(\int_{H W\left(G, \Delta^{+}\right)}^{\oplus} \pi_{\lambda}^{*} \otimes \pi_{\lambda} d \mu(\lambda), \int_{H W\left(G, \Delta^{+}\right)}^{\oplus} \mathscr{H}_{\lambda}^{*} \widehat{\otimes} \mathscr{H}_{\lambda} d \mu(\lambda)\right),
$$

where $\left(\pi_{\lambda}, \mathscr{H}_{\lambda}\right)$ is a unitary highest weight representation of $G$ with highest weight $\lambda$.

\section{Conuclear Spaces and Integral Representations}

The objective of this section is to explain all the concepts needed for Thomas' Integral Representation Theorem and eventually to state it.

Definition 1.1. Let $E$ be a topological vector space.

(a) We call a system $\mathfrak{S}$ of subsets of $E$ saturated

$$
\begin{aligned}
& (\forall A \in \mathfrak{S})(\forall t>0)(\exists B \in \mathfrak{S}) \quad t A \subseteq B, \\
& (\forall A, B \in \mathfrak{S})(\exists C \in \mathfrak{S}) \quad A \cup B \subseteq C .
\end{aligned}
$$

We denote the saturated system of all bounded convex closed balanced subsets of $E$ by $\widetilde{S}_{b c}$.

(b) Let $A \in \mathfrak{S}_{b c}$ and $E_{A}:=\cup_{t>0} t A$. Further we set

$$
p_{A}: E_{A} \rightarrow[0, \infty[, x \mapsto \inf \{t>0: x \in t A\} .
$$

Then $p_{A}$ is a norm on $E_{A}$. We consider $E_{A}$ as a topological vector space with the topology induced from $p_{A}$.

(c) Let $\mathscr{T}$ be the topology of $E$ and $\mathscr{T}_{c} \subseteq \mathscr{T}$ the subsystem of all convex balanced open sets. For each $U \in \mathscr{T}_{c}$ we define the seminorm

$$
q_{U}: E \rightarrow\left[0, \infty\left[; q_{U}(x):=\inf \{\mathrm{t}>0: x \in t U\}\right.\right.
$$

and put $N_{U}:=q_{U}^{-1}(\{0\})$. Then $q_{U}$ gives rise to a norm on $\mathrm{E}_{U}:=E / N_{U}$, also denoted by $q_{U}$.

(d) We write $E^{\prime}$ for the topological dual of $E$ and $E_{b}^{\prime}, E_{\sigma}^{\prime}$, resp. $E_{\tau}^{\prime}$, for $E^{\prime}$ equipped with the strong topology, weak-*-topology, resp. Mackey topology. 
(e) The completion of a topological vector space $E$ is denoted by $\widehat{E}$.

(f) A topological vector space $E$ is called quasicomplete if every bounded closed subset of $E$ is complete.

Remark I.2. (a) For all $A, B \in \widetilde{S}_{b c}, A \subseteq B$, one has continuous inclusions $E_{A} \rightarrow E_{B} \rightarrow E$. Moreover, $E_{A}$ is complete if $A \subseteq E$ is complete (cf. [Tr67, Lemma $36,1])$.

(b) If $U, V \in \mathscr{T}_{c}, U \subseteq V$, then one has continuous maps $E \rightarrow E_{U} \rightarrow E_{V}$.

Definition I.3. (a) A locally convex space $E$ is called nuclear if for each $U \in \mathscr{T}_{c}$ there exists $V \in \mathscr{T}_{c}, V \subseteq U$, such that $\widehat{E_{V}} \rightarrow \widehat{E_{U}}$ is nuclear.

(b) Let $\mathfrak{S}$ be a saturated subsystem of $\mathfrak{S}_{b c}$. A locally convex space $E$ is called S-conuclear, if for each $A \in \mathfrak{S}$ there exists $B \in \mathfrak{S}, A \subseteq B$, such that $\widehat{E_{A}} \rightarrow \widehat{E_{B}}$ is nuclear. We call $E$ conuclear if $E$ is $\widetilde{S}_{b c}$-conuclear.

The next proposition describes the interplay between nuclear and conuclear spaces.

Proposition I.4. ( ( ) Let E be a quasicomplete locally convex space. Then $E$ is conuclear if and only if its strong dual $E_{b}^{\prime}$ is nuclear.

(ii) The strong dual of a nuclear Fréchet space is nuclear.

(iii) Every nuclear Fréchet space is conuclear.

Proof. (1) [Sch 73, Ch. IV, Th. 1].

(ii) $[$ Tr67, Prop. 50.6]

(iii) Let $E$ be a nuclear Fréchet space. According to (ii), $E_{b}^{\prime}$ is nuclear. Thus (i) implies that $E$ is conuclear.

Example I.5. (a) Let $M$ be a finite dimensional separable complex manifold and $\mathrm{Hol}(M)$ the space of holomorphic functions on $M$ equipped with the topology of compact convergence. Then $\operatorname{Hol}(M)$ is a nuclear Fréchet space (cf. [Gr55, p. 56]) hence conuclear by Proposition I.4 (iii).

(b) More generally, let $E$ be a complete locally convex space over the complex numbers and $\operatorname{Hol}(M, E)$ the space of $E$-valued holomorphic functions on $M$ endowed with the topology of compact convergence. Then $\operatorname{Hol}(M, E)$ is complete and $\operatorname{Hol}(M, E) \cong \operatorname{Hol}(M) \widehat{\otimes}_{\varepsilon} E$ by $[G r 55$, Th. 13]. Thus $\operatorname{Hol}(M, E)$ is nuclear since Hol $(M)$ is nuclear (cf. [Tr67, Prop. 50.1 (9)]). If in addition $E$ is a Fréchet space, then $\operatorname{Hol}(M, E)$ is a nuclear Fréchet space and so conuclear by Proposition I.4 (iii). 
Definition $\mathbf{1}$. 6. Let $E$ be a topological vector space and $C \subseteq E$ a cone.

(a) The edge of $C$ is defined by $H(C):=C \cap(-C)$. We call $C$ pointed if $H(C)=$ $\{0\}$. Note that $H(C)$ is a vector space if $C$ is convex.

(b) If $C$ is convex, then we define an ordering on $C$ by $y \leq x: \Longleftrightarrow x-y \in C$. The cone $C$ is called a lattice if any two members of $C$ have a supremum with respect to this order relation. For each $x \in C$ we define the order interval $I_{x}:=C$ $\cap(x-C)=\{y \in C: y \leq x\}$.

(c) Let $\mathbb{R}^{+}:=[0, \infty[$. A subset $S \subseteq C \backslash\{0\}$ is called a section if $S$ meets each ray in $C$ in exactly one point, i.e., $\left|S \cap \mathbb{R}^{+} x\right|=1$ for each $x \in C \backslash\{0\}$. To each section $S$ we associate the function $p s: C \rightarrow \mathbb{R}^{+}$defined by $x \in p s(x)$. S. Note that $p_{s}$ is positively homogeneous and $S=\{x \in C$ : $p s(x)=1\}$.

(d) A Radon measure on a Hausdorff space $T$ is a locally finite inner regular Borel measure on $T$. We write $\mathscr{R}(T)$ for the cone of Radon measures on $T$.

(e) A section $S$ of $C$ is called admissible if $p_{s}: C \rightarrow \mathbb{R}^{+}$is universally measurable (cf. [Sch73, Ch. I] for the notion of universally measurable maps). An admissible parametrization of $C$ is an injective Borel map $\gamma: T \rightarrow C$ such that $\operatorname{im} \gamma \subseteq C$ is an admissible section, $\gamma^{-1}: \operatorname{im} \gamma \rightarrow T$ is universally measurable and $\left(\gamma^{-1}\right) *(\mathscr{R}(\mathrm{im} \gamma)) \subseteq \mathscr{R}(T)$.

(f) For each $x \in C$ we define the face generated by $x F_{x}:=\cup_{\lambda>0} I_{\lambda x}$. A point $x \in C$ is called an extreme generator if $F_{x}=\mathbb{R}^{+} x$. The set of extreme generators of $C$ is denoted by $\operatorname{Ext}(C)$. Note that $\operatorname{Ext}(C)$ is a not necessarily convex subcone of $C$.

Remark I.7. Our definition of an admissible parametrization is more general than the one given in [Th94]. There an admissible parametrization is defined to be an injective continuous map $\gamma: T \rightarrow C$ such that $\gamma$ is an admissible section and $\gamma^{-1}: \operatorname{im} \gamma \rightarrow T$ is universally measurable. We are lead to this generalization, since the parametrizations we have in mind are not continuous. However, as we will see below, the Integral Representation Theorem in [Th94, p. 226] remains valid with our notion of an admissible parametrization.

Note that there always exists an admissible parametrization of $C$ if $E$ is locally convex (cf. [Th94, Cor. 1.17]).

Now we have all notation to state Thomas' Integral Representation Theorem.

Theorem I.8. Let $E$ be a quasicomplete conuclear space and $C \subseteq E$ a closed convex cone such that all order intervals $I_{x}, x \in C$, are bounded. Let $\gamma: T \rightarrow \operatorname{Ext}(C)$ be an admissible parametrization of the cone of extremal generators.

(i) For each $x \in C$ there exists a Radon measure $\mu$ on $T$ such that $x=\int_{T} \gamma(t) d \mu(t)$ holds.

(ii) The measure $\mu$ is uniquely determined by $x$ if and only if $F_{x}$ is a lattice. In 
particular, $\mu$ is uniquely determined for all $x \in C$ if and only if $C$ is a lattice.

(iii) Let $\mathscr{R}(T)_{C}:=\left\{\mu \in \mathscr{R}(T): \int_{T} \gamma(t) d \mu(t) \in C\right\}$. Then $\mathscr{R}(T)_{C}$ is a convex subcone of $\mathscr{R}(T)$ and the order preserving map

$$
\Phi:\left(\mathscr{R}(T)_{c}, \leq\right) \rightarrow(C, \leq), \mu \mapsto \int_{T} \gamma(t) d \mu(t)
$$

is onto; it is bijective if and only if $C$ is a lattice.

(iv) Let $\mu \in \mathscr{R}(T)_{C}$ and $x=\int_{T} \gamma(t) d \mu(t) \in C$. Let $F_{\mu}$ be the face generated by $\mu$ in $\mathscr{R}(T)$. Then $F_{\mu} \subseteq R(T)_{c}$ and $\Phi$ induces an order preserving map $\Phi_{\mu}:\left(F_{\mu}, \leq\right) \rightarrow\left(F_{x}\right.$, $\leq)$. Moreover, $\Phi_{\mu}$ is an order isomorphism if and only if $F_{x}$ is a lattice.

Proof. (i), (ii) Fix $x \in C$. By the definition of an admissible parametrization $S:=i m \gamma$ is an admissible section, so that [Th94, Th.1.18, Th.5.2] imply that there exists a Radon measure $\nu$ on $S$ such that $x=\int_{S} y d \nu(y)$, and, moreover, $\nu$ is unique if and only if $F_{x}$ is a lattice.

Again by the definition of an admissible parametrization $\mu:=\left(\gamma^{-1}\right) * . \nu$ is a Radon measure on $T$. Thus we obtain that $x=\int_{T} \gamma(t) d \mu(t)$, and $\mu$ is unique if and only if $F_{x}$ is a lattice.

(iii) This is a direct consequence of (1) and (ii).

(iv) It is clear that $F_{\mu} \subseteq \mathscr{R}(T)_{C}$ and hence $\Phi_{\mu}$ is a well define order preserving map.

It follows from the Radon-Nikodym Theorem (cf. [Sch73, Ch.I, §6, Th. 14]) that $\left(F_{\mu}, \leq\right)$ is a lattice. Thus $F_{x}$ is a lattice whenever $\Phi_{\mu}$ is an order isomorphism.

To prove the converse, let $\mu$ be uniquely determined by $x$. In view of (ii), this means that $F_{x}$ is a lattice. We claim $F_{y}$ is a lattice for every $y \in F_{x}$. In fact, let $a, b \in F_{y}$. Since $F_{x}$ is a lattice, $a \vee b$ and $a \wedge b$ exists in $F_{x}$. Now $a \vee b, a \wedge b \in F_{y}$, proving the claim.

According to (ii), our claim implies that every element of $F_{x}$ has a unique measure representing it. In particular, $\Phi_{\mu}$ is injective. Next we show that $\Phi_{\mu}$ is onto. Fix $y \in F_{x}$ and let $\nu \in \mathscr{R}(T)_{c}$ be a corresponding measure. W.1.o.g. we may assume that $y \leq x$. Then $x-y \in C$ and we find a Radon measure $\sigma$ representing $x-y$. But then $\tilde{\mu}=\nu+\sigma$ is a Radon measure representing $x$, hence equal to $\mu$ by the uniqueness of $\mu$. Thus $\nu \leq \mu$, and so $\nu \in F_{\mu}$. This shows that $\Phi_{\mu}$ is onto and also that $\Phi_{\mu}^{-1}$ is order preserving, concluding the proof of (iv).

\section{Positive Definite Kernels on Complex Manifolds}

In this section $M$ denotes always a complex manifold and we write $\bar{M}$ for $M$ equipped with the opposite complex structure. It is our aim to apply the Integral Representation Theorem to the conuclear space Hol $(M \times \bar{M}, V)$ and closed convex subcones of the cone $\mathscr{P}\left(M^{2}, V\right)$ of positive definite holomorphic $B(V)$ - 
valued kernels.

Definition II.1. (a) Let $X$ be a set, $V$ a Hilbert space and $B(V)$ the space of bounded operators on $V$. A function $K: X \times X \rightarrow B(V)$ is called $B(V)$-valued positive definite kemel if for every sequence $\left(x_{1}, v_{1}\right), \ldots,\left(x_{n}, v_{n}\right)$ in $X \times V$ we have

$$
\sum_{j, k=1}^{n}\left\langle K\left(x_{j}, x_{k}\right) v_{k}, v_{j}\right\rangle \geq 0 .
$$

We write $\mathscr{P}\left(X^{2}, V\right)$ for the set of all $B(V)$-valued positive definite kernels.

If $V \cong \mathbb{C}$ is one-dimentional, then the elements $K \in \mathscr{P}\left(X^{2}\right):=\mathscr{P}\left(X^{2}, V\right)$ are simply called positive definite kernels on $X$.

(b) If $X=M$ is a complex manifold, then we call $K \in \mathscr{P}\left(M^{2}, V\right)$ a $B(V)$-valued holomorphic positive definite kermel if $K$ is positive definite and $K \in \operatorname{Hol}(M \times \bar{M}$, $B(V))$.

Proposition II.2. Let $X$ be a set and $V$ a Hilbert space.

(1) If $K \in \mathscr{P}\left(X^{2}, V\right)$, then $K(x, x)$ is a positive semidefinite operator and $K(x, y)$ * $=K(y, x)$ holds for all $x, y \in X$.

(ii) A function $K: X \times X \rightarrow B(V)$ is a $B(V)$-valued positive definite kemel if and only if there exists a Hilbert space $\mathscr{H} \subseteq V^{X}$ with continuous point evaluations $K_{x}$ : $\mathscr{H}$ $\rightarrow V, f \mapsto f(x)$ such that $K(x, y)=K_{x} K_{y}^{*}$ holds for all $(x, y) \in X \times X$. In this case we write $\mathscr{H}_{K}$ for $\mathscr{H}$.

(iii) If $K \in \mathscr{P}\left(X^{2}, V\right)$, then

$$
\mathscr{H}_{K}^{0}:=\operatorname{span}\left\{K_{x, v}: x \in X, v \in V\right\} \subseteq \mathscr{H}_{K},
$$

where $K_{x, v}(y):=K(y, x) . v$, is a dense subspace of $\mathscr{H}_{K}$.

(iv) If $X=M$ is a complex manifold and $K \in \mathscr{P}\left(M^{2}, V\right)$, then $\mathscr{H}_{K} \subseteq \mathrm{Hol}(M, V)$ consists of holomorphic functions.

Proof. (i) [Ne99, Lemma I. 2].

(ii), (iii) [Ne99, Th. I. 4].

(iv) [Ne99, Prop. IV. 1. 9].

We refer to $\mathscr{H}_{K}$ as a reproducing kemel Hilbert space corresponding to the positive definite $B(V)$-valued kernel $K$.

From now on $V$ always denotes a Hilbert space and $S$ an involutive semigroup.

Proposition II.3. Let $M$ be a complex manifold and $\mathrm{Hol}(M \times \bar{M}, V)$ be equipped with the topology of compact convergence. Then the following assertions hold:

(i) The cone $\mathscr{P}\left(M^{2}, V\right)$ is closed and convex in $\operatorname{Hol}(M \times \bar{M}, V)$. 
(ii) All order intervals $I_{K}, K \in \mathscr{P}\left(M^{2}, V\right)$, are closed and bounded.

(iii) If $M$ is separable and $V$ is finite dimensional, then all order intervals $I_{K}, K \in$ $\mathscr{P}\left(M^{2}, V\right)$, are compact.

Proof. (i) This is clear.

(ii) That order intervals are closed is obvious. To show that they are bounded we note that the topology on $\operatorname{Hol}(M \times \bar{M}, V)$ is induced from the seminorms

$$
q_{A}(f):=\sup _{x, y \in A}\|f(x, y)\|,
$$

where $A$ runs over all compact subsets of $M$. We show that

$$
\left(\forall K \in \mathscr{P}\left(M^{2}, V\right)\right) q_{A}(K)=\sup _{x \in A}\|K(x, x)\| .
$$

The inequality " $\geq$ " is clear. Using Proposition II.2(ii), we obtain that

$$
\|K(x, y)\| \leq\left\|K_{x}\right\| \cdot\left\|K_{y}\right\|=\sqrt{K(x, x)} \sqrt{K(y, y)}
$$

for all $x, y \in M$, proving the converse.

Now we can show that $I_{K}$ is bounded. Let $Q \in I_{K}$. Then $Q \leq K$ implies that $K(x, x)-Q(x, x)$ is positive definite for all $x \in M$, and so

$$
\left(\forall Q \in I_{K}\right)(\forall x \in M)\|Q(x, x)\| \leq\|K(x, x)\| .
$$

Equations (2.1) and (2.3) imply for all $Q \in I_{K}$ and all the seminorms $q_{A}$ that

$$
q_{A}(Q)=\sup _{x \in M}\|Q(x, x)\| \leq \sup _{x \in A}\|K(x, x)\|=q_{A}(K),
$$

i.e., $I_{K}$ is bounded.

(iii) Note that $\operatorname{Hol}(M \times \bar{M}, V)$ is a nuclear Fréchet space, provided $M$ is separable and $V$ is finite dimensional (cf. Example I.5 (b)). Therefore $\operatorname{Hol}(M \times \bar{M}, V)$ is a Montel space (cf. [Tr67, Prop. 50.2]). In particular, closed and bounded sets are compact. In view of (ii), this proves the assertion.

Proposition II.4. Let $M$ be a separable complex manifold and $V$ a finite dimensional Hilbert space. Let $C$ be a closed convex subcone of $\mathscr{P}\left(M^{2}, V\right)$ and $\gamma: T$ $\rightarrow$ Ext $C$ be an admissible parametrization. Set $K^{t}:=\gamma(t)$ for all $t \in T$.

(i) For each $K \in C$ there exists a Radon measure $\mu$ on $T$ such that $K=\int_{T} K^{t} d \mu(t)$

(ii) The measure $\mu$ is unique if and only if $F_{K}$ is a lattice.

Proof. According to Example I.5 (b), the topological vector space $\mathrm{Hol}(M \times$ $\bar{M}, V)$ is quasicomplete and conuclear. Moreover Proposition II.3 implies that $\mathscr{P}\left(M^{2}, V\right)$ is a closed convex cone in $\operatorname{Hol}(M \times \bar{M}, V)$ such that all order intervals $I_{K}$ are compact. As $C$ is a closed convex subcone of $\mathscr{P}\left(M^{2}, V\right)$, the same holds 
for $C$. Thus all assumptions of Theorem 1.8 are satisfied and the assertion follows.

For each Hilbert space $\mathscr{H}$ we write Herm $(\mathscr{H})+$ for the convex cone of positive operators in $B(\mathscr{P})$. Let $K \in \mathscr{H}\left(M^{2}, V\right)$ and $\mathscr{H}_{K}$ be the corresponding Hilbert space (cf. Proposition II.2 (iv)). To each $A \in \operatorname{Herm}\left(\mathscr{H}_{K}\right)^{+}$we associate an element $K_{A} \in \mathscr{P}\left(M^{2}, V\right)$ by the prescription $K_{A}(x, y):=K_{x} A K_{y}^{*}$ for all $x, y \in M$ (cf. [Ne99, Lemma I.7 (ii)]). If $C$ is a closed convex subcone in $\mathscr{P}\left(M^{2}, V\right)$ we, set

$$
\mathscr{A}^{+}(K, C):=\left\{A \in \operatorname{Herm}\left(\mathscr{H}_{K}\right)^{+}: K_{A} \in C\right\} .
$$

Lemma II.5. Suppose that $V$ is finite dimensional and let $C \subset \mathscr{P}\left(M^{2}, V\right)$ be a closed convex subcone. Let $K \in C, K=\int_{T} K^{t} d \mu(t)$ be an integral representation of $K$ with respect to $C$ and $F_{K}$ the face generated by $K$ in $C$.

(i) The mapping $\Psi_{K}:\left(\mathscr{A}^{+}(K, C), \leq\right) \rightarrow\left(F_{K}, \leq\right), A \mapsto K_{A}$ is an order isomorphism.

(ii) Let $\Phi_{\mu}:\left(F_{\mu}, \leq\right) \rightarrow\left(F_{K}, \leq\right)$ be the map of Theorem I.8(iv). Then $\Psi_{K}^{-1} \circ \Phi_{\mu}$ : $\left(F_{\mu}, \leq\right) \rightarrow\left(\mathscr{A}^{+}(K, C), \leq\right)$ is an order isomorphism if and only if $\mu$ is uniquely determined by $K$.

(iii) Let $\mathscr{B}$ be the Borel $\sigma$-algebra of $T$ and set $K^{B}:=\int_{B} K^{t} d \mu(t)$ for each $B \in \mathscr{B}$. Then $K^{B} \in C, K^{B} \leq K$ and $\mathscr{H}_{K^{B}} \subseteq \mathscr{H}_{K}$. Moreover, if $\mu$ is unique, then the inclusion mapping $i_{B}: \mathscr{H}_{K B} \rightarrow \mathscr{H}_{K}$ is isometric for all $B \in \mathscr{B}$.

Proof. (1) This follows from [Ne99, Th.I.20].

(ii) This is a consequence of Theorem I.8 and (i).

(iii) Since $K^{B}=\Phi_{\mu}\left(\mathbb{1}_{B} d \mu\right)$ and $\mathbb{1}_{B} d \mu \in F_{\mu}$, it follows from Theorem I.8(iv) that $K^{B}$ $\leq K$ and $K^{B} \in C$ for all $B \in \mathscr{B}$. As $K^{B} \leq K$, [Ne99, Th. I. 20] implies that $\mathscr{H}_{K^{B}}$ is continuously included in $\mathscr{H}_{K}$.

To prove the second assertion we first note that $i_{B}$ is isometric if and only if $\mathscr{H}_{K^{B}} \oplus \mathscr{H}_{K^{B}}=\mathscr{H}_{K}$ is an orthogonal direct sum. Write $A_{B}:=\left(\Psi_{K}^{-1} \circ \Phi_{\mu}\right)\left(\mathbb{1}_{B} d \mu\right)$ for $B \in \mathfrak{B}$. If $\mu$ is unique, then (ii) and $\inf \left\{\mathbb{1}_{B} d \mu, \mathbb{1}_{B} c d \mu\right\}=0$ imply that $\operatorname{im} A_{B} \perp$ $\operatorname{im} A_{B c}$, i.e., $\mathscr{H}_{K^{B}} \oplus \mathscr{H}_{K^{B}}=\mathscr{H}_{K}$ for all $B \in \mathfrak{B}$, as was to be shown.

Remark II.6. The converse of Lemma II.5(iii) is false, i.e., $i_{B}$ isometric for all $B \in \mathscr{B}$ does not imply that $\mu$ is unique. In fact let $M=\{x\}, V$ be a finite dimensional Hilbert space of dimension $n \geq 2$ and $K \in \mathscr{P}\left(M^{2}, V\right)$ defined by $K(x, x)=\mathrm{id} v$. For each $v \in V$ we write $P_{v}: V \rightarrow \mathbb{C} . v$ for the orthogonal projection onto $[v]:=\mathbb{C} . v$ and denote by $\mathbb{P}(V):=\{[v]: v \in V \backslash\{0\}\}$ the projective space of $V$. Then $\operatorname{Ext}\left(\mathscr{P}\left(M^{2}, V\right)\right)=\left\{P_{v}:[v] \in \mathbb{P}(V)\right\}$ and

$$
\gamma: \mathbb{P}(V) \rightarrow \operatorname{Ext}\left(\mathscr{P}\left(M^{2}, V\right)\right),[v] \mapsto \frac{1}{\langle v, v\rangle} P_{v}
$$


is a continuous bijection of $\mathbb{P}(V)$ onto a section of $\operatorname{Ext}\left(\mathscr{P}\left(M^{2}, V\right)\right)$, hence an admissible parametrization.

Every othornormal basis $\left\{v_{1}, \ldots, v_{n}\right\}$ of $V$ gives via $K=\sum_{j_{=1}}^{n} P_{v j}$ rise to a measure $\mu_{v_{1}, \ldots, v n}:=\sum_{j=1}^{n} \delta_{[v j]}$ representing $K$. In particular, we see that $\mu$ is not unique.

Now fix a measure $\mu_{v_{1}, \cdots, v_{n}}$ representing $K$ corresponding to the orthonormal basis $\left\{v_{1}, \ldots, v_{n}\right\}$. Then

$$
\left\{K^{B}: B \in \mathscr{B}(S(V))\right\}=\left\{\sum_{j \in J} P_{v}: J \subseteq\{1, \ldots, n\}\right\} .
$$

Therefore, if $K^{B}=\sum_{j \in J} P_{v}$, one has $\mathscr{H}_{K^{B}}=\operatorname{span}\left\{v_{j}: j \in J\right\}$ and the inclusion mapping $i_{B}: \mathscr{H}_{K^{B}} \rightarrow \mathscr{H}_{K}=V$ is isometric.

Definition II.7. (cf. [Fo96, p.220]) Let $(T, \mathbb{S})$ be a measure space, i.e., $T$ is a set and $\mathfrak{S}$ in a $\sigma$-algebra on $T$. A measurable field of Hilbert spaces is a family of Hilbert spaces $\left(\mathscr{H}_{t}\right)_{t \in T}$ together with a countable set $\left\{x_{n}: n \in \mathbb{N}\right\} \subseteq$ $\prod_{t \in T} \mathscr{H}_{t}$ of sections such that the following axioms are satisfied:

(F1) The mappings $t \mapsto\left\langle x_{j}(t), x_{k}(t)\right\rangle_{t}$ are measurable for all $j, k \in \mathbb{N}$.

(F2) The linear span of $\left\{x_{n}(t): n \in \mathbb{N}\right\}$ is dense in $\mathscr{H}_{t}$ for all $t \in T$.

If, in addition, $\mu$ is a measure on $(T, \mathfrak{S})$, then we denote the corresponding direct integral of Hilbert spaces by $\int_{T}^{\oplus} \mathscr{H}_{t} d \mu(t)$.

Proposition II.8. Let $M$ be a separable complex manifold, $V$ a finite dimensional Hilbert space, $C$ a closed convex subcone of $\mathscr{P}\left(M^{2}, V\right)$ and $\gamma: T \rightarrow \operatorname{Ext} C$, $t \mapsto K^{t}$, an admissible parametrization. Let $x \in C$ and $\mu$ a Radon measure on $T$ representing $x$. We set $\mathscr{H}_{t}:=\mathscr{H}_{K^{t}}$ for all $t \in T$.

(1) Let $\left\{\left(x_{n}, v_{n}\right) \in M \times V: n \in \mathbb{N}\right\}$ be a dense subset in $M \times V$ and set $x_{n}(t):=$ $K^{t}{ }_{x, v_{n}}$ for all $n \in \mathbb{N}, t \in T$. Then $\left\{x_{n}: n \in \mathbb{N}\right\}_{\text {induces on }}\left(\mathscr{H}_{t}\right)_{t \in T}$ the structure of $a$ Borel measurable field of Hilbert spaces. We write $\int_{T}^{\oplus} \mathscr{H}_{t} d \mu(t)$ for the direct integral corresponding to the Radon measure $\mu$.

(ii) The mapping $\Omega_{T}: \int_{T}^{\oplus} \mathscr{H}_{t} d \mu(t) \rightarrow \mathscr{H}_{K}$ given by $\left\langle\Omega_{T}(f)(x), v\right\rangle:=\left\langle f,\left(K^{t}{ }_{x, v}\right)_{t \in T}\right\rangle$ yields an isometry of $\left(\operatorname{ker} \Omega_{T}\right)^{\perp} \rightarrow \mathscr{H}_{K}$. Moreover, if $\mu$ is unique, then $\Omega_{T}$ is an isomorphism.

Proof. (i) To prove (F्F1) it suffices to check that the mapping $\varphi_{y, x, w, v}: T \rightarrow$ $\mathbb{C}, t \mapsto\left\langle K_{x, v}^{t}, K_{y, w}^{t}\right\rangle_{t}$ are Borel measurable for $x, y \in M, v, w \in V$. As $\gamma: T \rightarrow \operatorname{Ext}(C)$ is an admissible parametrization, it is Borel measurable by definition. Moreover, for all $x, y \in M, v, w \in V$, the evaluation mapping

$$
\operatorname{ev}_{y, x, w, v}: \operatorname{Hol}(M, \times \bar{M}, B(V)) \rightarrow \mathbb{C}, f \mapsto\langle f(y, x) \cdot w, v\rangle
$$

is continuous. Thus $\varphi_{y, x, w, v}=\mathrm{ev}_{y, x, w, v} \circ \gamma$ is the composition of two Borel 
measurable mappings, hence Borel measurable. This proves ( $\mathscr{F} 1)$.

Since $(\mathscr{F} 2)$ is obviously satisfied, the assertion follows.

(ii) For every $x \in M$ and $B \in \mathscr{B}$ we define a linear map

$$
\eta_{B}(x): V \rightarrow \int_{B}^{\oplus} \mathscr{H}_{t} d \mu(t), v \mapsto\left(K_{x, v}^{t}\right)_{t \in B}
$$

We have

$$
\begin{aligned}
\left\langle\eta_{B}(x)(v), \eta_{B}(y)(w)\right\rangle & =\int_{B}\left\langle K_{x, v}^{t}, K_{y, w}^{t}\right\rangle d \mu(t)=\int_{B}\left\langle K^{t}(y, x) . v, w\right\rangle d \mu(t) \\
& =\left\langle\int_{B} K^{t}(y, x) d \mu(t) . v, w\right\rangle \\
& =\left\langle K^{B}(y, x) . v, w\right\rangle \leq\left\|K^{B}(y, x)\right\| \cdot\|v\| \cdot\|w\|
\end{aligned}
$$

for all $x, y \in M, v, w \in V$. This shows in particular that all $\eta_{B}(x)$ are bounded operators and hence the well-definedness of the mapping

$$
\eta_{B}: M \rightarrow B\left(V, \int_{B}{ }^{\oplus} \mathscr{H}_{t} d \mu(t)\right), x \mapsto \eta_{B}(x) .
$$

Moreover, the computation also shows that $K^{B}(x, y)=\eta_{B}(x) * \eta_{B}(y)$ so that the Realization Theorem (cf. [Ne99,Th.I.11]) implies that the mapping

$$
\Omega_{B}: \int_{B}^{\oplus} \mathscr{H}_{t} d \mu(t) \rightarrow \mathscr{H}_{K^{B}}, \Omega_{B}(f)(x)=\eta_{B}(x)^{*} . f
$$

defines an isometry of $\left(\operatorname{ker} \Omega_{B}\right){ }^{\perp}$ onto $\mathscr{H}_{K B}$. This shows the first assertion.

Finally we have to show that $\Omega$ is an isometry if $\mu$ is uniquely determined by $K$. To each $B \in \mathscr{B}$ we associate the projection $P_{B}: \int_{T}^{\oplus} \mathscr{H}_{t} d \mu(t) \rightarrow \int_{B}^{\oplus} \mathscr{H}_{t} d \mu(t)$, $\left(f_{t}\right)_{t \in T} \mapsto\left(f_{t}\right)_{t \in B}$. It follows from $\eta_{B}=P_{B} \circ \eta$ that $i_{B} \circ \Omega_{B}=\Omega_{T} \circ P_{B}^{*}$, i.e., the diagram

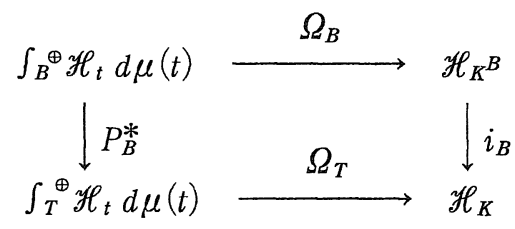

is commutative. Taking adjoints we obtain that

$$
P_{B} \circ \Omega_{T}^{*}=\Omega_{B}^{*} \circ i_{B}^{*}
$$

Assume now that $\mu$ is uniquely determined by $K$. Then Lemma II.5 (iii) implies that $i_{B}$ is isometric for all $B \in \mathscr{B}$. Thus it follows from (2.4) and the isometry of $\Omega_{B}^{*}$ and $\Omega_{T}^{*}$ that $\left.P_{B}\right|_{\Omega_{T}^{*}\left(\mathscr{H}_{K}^{B)}\right.}$ is isometric and $\left.P_{B}\right|_{\Omega_{T}^{*}\left(\mathscr{H}_{K} K^{B C}\right)}=0$ for all $B$ $\in \mathscr{B}$. Since $\mathscr{H}_{K}=\mathscr{H}_{K^{B}} \oplus \mathscr{H}_{K^{B}}$ for all $B \in \mathscr{B}$, this implies that $\operatorname{im} \Omega_{T}^{*}$ is invariant under $\left\{P_{B}: B \in \mathscr{B}\right\}$, i.e., $\operatorname{im} \Omega_{T}^{*}$ is a decomposable subspace of $\int_{T}^{\oplus} \mathscr{H}_{t} d \mu(t)$. In view of $[\mathrm{Fo} 96,7.29]$ this shows that $\Omega_{T}$ is onto, proving the second assertion. 


\section{Application to Representation Theory}

Definition II.9. (a) An involutive semigroup is a semigroup $S$ together with an involutive antiautomorphism $*: S \rightarrow S, s \mapsto s^{*}$.

(b) Let $X$ be a set and $S$ an involutive semigroup acting on $X$ from the left. An element $K \in \mathscr{P}\left(X^{2}, V\right)$ is called $S$-invariant, or simply invariant, if $K(s . x, y)=$ $K\left(x, s^{*} . y\right)$ holds for $x, y \in X, s \in S$. We write $\mathscr{P}\left(X^{2}, V\right)_{i}$ for the subcone of all invariant elements of $\mathscr{P}\left(X^{2}, V\right)$.

(c) Let $K \in \mathscr{P}^{2}\left(X^{2}, V\right)_{i}$. Then

$$
\pi_{K}^{0}: S \rightarrow \operatorname{End}\left(\mathscr{H}_{K}^{0}\right),\left(\pi_{K}^{0}(s) . f\right)(x)=f\left(s^{*} . x\right)
$$

defines a semigroup homomorphism. Note that all operators $\pi_{K}^{0}(s), s \in S$, are closable on $\mathscr{H}_{K}$ (cf. [Ne99, Prop. II. 4. 10]) and that $\pi_{K}^{0}\left(s^{*}\right)=\left.\pi_{K}^{0}(s) *\right|_{\mathscr{H}_{k}^{0}}$ holds for all $s \in S$. We call $\left(\pi_{K}^{0}, \mathscr{H}_{K}^{0}\right)$ a hermitian representation of $S$.

Further, $\pi_{K}^{0}(s)$ is bounded if and only if

$$
\begin{aligned}
\alpha_{K}(s):=\left\|\pi_{K}^{0}(s)\right\|^{2}=\sup _{\substack{x \in M, v \in V \\
K_{x, v \neq 0}}} \frac{\left\langle\pi_{K}^{0}(s) . K_{x, v}, \pi_{K}^{0}(s) . K_{x, v}\right\rangle}{\left\langle K_{x, v}, K_{x, v}\right\rangle} \\
=\sup _{\substack{x \in M, v \in V \\
K_{x, v \neq 0}}} \frac{\left\langle K_{s . x, v}, K_{s . x, v}\right\rangle}{\left\langle K_{x, v}, K_{x, v}\right\rangle}=\sup _{\substack{x \in M, v \in V \\
K_{x, v \neq 0}}} \frac{\langle K(s . x, s . x) \cdot v, v\rangle}{\langle K(x, x) \cdot v, v\rangle}<\infty
\end{aligned}
$$

(cf. [Ne99, Th. II. 4. 4]). If $\alpha_{K}(s)$ is finite for all $s \in S$, then $\left(\pi_{K}^{0}, \mathscr{H}_{K}^{0}\right)$ extends to a representation $\left(\pi_{K}, \mathscr{H}_{K}\right)$ of $S$ given by

$$
\pi_{K}: S \rightarrow B\left(\mathscr{H}_{K}\right),\left(\pi_{K}(s) . f\right)(x)=f\left(s^{*} \cdot x\right),
$$

i.e., $\pi_{K}$ is a semigroup homomorphism and $\pi_{K}\left(s^{*}\right)=\pi_{K}(s) *$ holds for all $s \in S$ (cf. [Ne99, Th. III. 1. 3]).

(d) A mapping $\alpha: S \rightarrow \mathbb{R}^{+}$is called an absolute value of $S$ if $\alpha(s)=\alpha\left(s^{*}\right)$ and $\alpha(s t) \leq \alpha(s) \alpha(t)$ holds for all $s, t \in S$. To each absolute value $\alpha$ we associate a subset of $\mathscr{P}\left(X^{2}, V\right)_{i}$ by

$$
\mathscr{P}\left(X^{2}, V, \alpha\right)_{i}:=\left\{K \in \mathscr{P}\left(X^{2}, V\right)_{i}: \alpha_{K} \leq \alpha\right\} .
$$

(e) A representation $(\pi, \mathscr{H})$ of $S$ is called multiplicity-free if its commutant $\pi(S)^{\prime}$ is abelian.

Lemma II.10. The set $\mathscr{P}\left(X^{2}, V, \alpha\right)_{i}$ is a convex subcone of $\mathscr{P}\left(X^{2}, V\right)_{i}$.

Proof. As $\mathscr{P}\left(X^{2}, V, \alpha\right)_{i}$ is invariant under multiplication with non-negative scalars, $\mathscr{P}\left(X^{2}, V, \alpha\right)_{i}$ is a cone.

We show that $\mathscr{P}\left(X^{2}, V, \alpha\right)_{i}$ is an additive semigroup. According to the 
Realization Theorem (cf. [Ne99, Th. I. 11, Rem. I. 12]), there exists an isometric $S$-equivariant embedding

$$
\left(\pi_{K_{1}+K_{2}}, \mathscr{H}_{K_{1}+K_{2}}\right) \rightarrow\left(\pi_{K_{1}} \oplus \pi_{K_{2}}, \mathscr{H}_{K_{1}} \oplus \mathscr{H}_{K_{2}}\right) .
$$

We conclude that $\alpha_{K_{1}+K_{2}} \leq \max \left(\alpha_{K_{1}}, \alpha_{K_{2}}\right) \leq \alpha$, proving the lemma.

In general it is far from being true that $F_{K}$ for $K \in \mathscr{P}\left(X^{2}, V, \alpha\right)_{i}$ is a lattice. But, as we will see below, in the important case where $\left(\pi_{K}, \mathscr{H}_{K}\right)$ is multiplicity-free this is true.

Proposition II.11. Let $K \in \mathscr{P}\left(X^{2}, V, \alpha\right)_{i}$. Then $F_{K}$ is a lattice if and only if $\left(\pi_{K}, \mathscr{H}_{K}\right)$ is multiplicity-free.

Proof. Recall from Lemma II.5(i) that the mapping

$$
\left(\mathscr{A}^{+}\left(K, \mathscr{P}\left(X^{2}, V, \alpha\right)_{i}\right), \leq\right) \rightarrow\left(F_{K}, \leq\right), A \mapsto K_{A}
$$

is an isomorphism of ordered spaces. Let $\mathscr{A}\left(K, \mathscr{P}\left(X^{2}, V, \alpha\right)_{i}\right) \subseteq B\left(\mathscr{H}_{K}\right)$ be the von Neumann algebra generated by $\mathscr{A}^{+}\left(K, \mathscr{P}\left(X^{2}, V, \alpha\right)_{i}\right)$. In view of $[\mathrm{Ne} 99$, Prop. II. 4. 29], we have

$$
\mathscr{A}^{+}\left(K, \mathscr{P}\left(X^{2}, V, \alpha\right)_{i}\right)=\left\{A \in \operatorname{Herm}\left(\mathscr{H}_{K}\right)^{+}: K_{A} \in \mathscr{P}\left(X^{2}, V, \alpha\right)_{i}\right\}=\left\{A \in \pi_{K}(S)^{\prime}: A \geq 0\right\},
$$

and hence $\mathscr{A}\left(K, \mathscr{P}\left(X^{2}, V, \alpha\right)_{i}\right)=\pi_{K}(S)^{\prime}$.

According to Sherman's Theorem (cf. [Th78, Lemma 8]), the positive cone of a von Neumann algebra is a lattice if and only if the algebra is abelian. In view of this fact, the proposition follows.

Theorem 11.12. Let $M$ be a separable complex manifold, $V$ a finite dimensional Hilbert space, $S$ an involutive semigroup acting on $M$ by holomorphic mappings from the left, $\alpha: S \rightarrow \mathbb{R}^{+}$an absolute value and $\gamma: T \rightarrow \operatorname{Ext}\left(\mathscr{P}\left(M^{2}, V, \alpha\right){ }_{i}\right)$, $t \mapsto K^{t}$ an admissible parametrization. Let $K \in \mathscr{P}\left(M^{2}, V, \alpha\right)_{i}$ and assume that $\left(\pi_{K}, \mathscr{H}_{K}\right)$ is multiplicity-free.

(i) The set $\mathscr{P}\left(M^{2}, V, \alpha\right)_{i}$ is a closed convex subcone of $\mathscr{P}\left(M^{2}, V\right)_{i}$ and the face $F_{K}$ is a lattice.

(ii) For each $Q \in F_{K}$ there exists a unique Radon measure $\mu$ on $T$ such that $Q=$ $\int_{T} K^{t} d \mu(t)$ holds.

(iii) All representations $\left(\pi_{t}^{0}, \mathscr{H}_{t}^{0}\right):=\left(\pi_{K^{t}}^{0}, \mathscr{H}_{K^{t}}^{0}\right), t \in T$, are $\alpha$-bounded, i.e., $\alpha_{t}(s):=$ $\left\|\pi_{t}^{0}(s)\right\| \leq \alpha(s)$ holds for all $s \in S$. In particular, each $\left(\pi_{t}^{0}, \mathscr{H}_{t}^{0}\right)$ extends to an involutive representation $\left(\pi_{t}, \mathscr{H}_{t}\right)$ of $S$.

(iv) For all $t \in T$ the representation $\left(\pi_{t}, \mathscr{H}_{t}\right)$ is irreducible.

(v) The prescription $K_{x, v} \mapsto\left(K_{x, v}^{t}\right)_{t \in T}$ defines a unitary equivalence

$$
\omega:\left(\pi_{K}, \mathscr{H}_{K}\right) \rightarrow\left(\int_{T}^{\oplus} \pi_{t} d \mu(t), \int_{T}^{\oplus} \mathscr{H}_{t} d \mu(t)\right)
$$


of $S$-modules.

Proof. (i) The first assertion follows from Lemma II.10, the second one from Proposition II.11.

(ii) This follows from (i) and Proposition II.4(i1).

(iii) As all $K^{t}, t \in T$, are contained in $\mathscr{P}\left(M^{2}, V, \alpha\right)_{i}$, the assertion is clear.

(iv) Since each $K^{t} \in \operatorname{Ext}\left(\mathscr{P}\left(M^{2}, V, \alpha\right)_{i}\right), t \in T$, is an extreme generator, the assertion follows from [Ne99, Cor. II. 4. 23].

(v) Using the notation from Proposition II.8(ii) we see that $\omega=\Omega_{T}^{*}$ and thus $\omega$ is well defined.

In view of Proposition II.8(ii) and (i), $\omega$ is in fact an isometric isomorphism. It remains to show that $\omega$ is $S$-equivariant. But this is a direct consequence of the $S$-equivariance of the mapping $\eta_{T}$, which follows from

$$
\eta_{T}(s . x)(v)=\left(K_{s . x, v}^{t}\right)_{t \in T}=\left(\pi_{K}^{t}(s) . K_{x, v}^{t}\right)_{t \in T}=\left(\int_{T}^{\oplus} \pi_{t}(s) d \mu(t)\right) . \eta_{T}(x)(v)
$$

for all $x \in M, v \in V$ and $s \in S$.

\section{Extension to the Envelope of Holomorphy}

We conclude this paragraph with a remark on the extension of a positive definite holomorphic kernel on a complex manifold $M$ to a positive definite holomorphic kernel on the envelope of holomorphy of $M$.

Definition II.13. (a) Let $M$ be a complex manifold. We write

$$
S(\operatorname{Hol}(M)):=\operatorname{Hom}_{\mathbb{C}}(\operatorname{Hol}(M), \mathbb{C})
$$

for the set of all continuous $\mathbb{C}$-algebra homomorphisms of $\mathrm{Hol}(M)$ to $\mathbb{C}$ endowed with the corresponding weak-*-topology. Then we have a continuous map

$$
\eta: M \rightarrow S(\operatorname{Hol}(M)), \eta(x)(f)=f(x) .
$$

Note that $\eta$ is in fact a homeomorphism if $M$ is a Stein manifold (cf. [Ro63, Th. 2.6]).

(b) Assume now that $M$ is a Stein manifold and let $D \subseteq M$ be a domain. According to [Re63, Th. 4.6], the space $\widehat{D}:=S(\mathrm{Hol}(D))$ carries the structure of a Stein manifold such that the canonical map

$$
\widehat{D} \rightarrow S(\operatorname{Hol}(M))=M, \varphi \mapsto\left(f \mapsto \varphi\left(\left.f\right|_{D}\right)\right)
$$

defines on $\widehat{D}$ the structure of a Riemann domain, i.e., $q$ is locally biholomorphic. The space $\widehat{D}$ is called the envelope of holomorphy of $D$. 
Proposition 11.14. Let $M$ be a Stein manifold, $D \subseteq M$ a domain, $\widehat{D}$ its envelope of holomorphy and $V$ a Hilbert space.

(i) The action of $S$ extends to an action $S \times \widehat{D} \rightarrow \widehat{D}$ by holomorphic maps.

(ii) Each $K \in \mathscr{P}\left(D^{2}, V\right)_{i}$ extends to an element $\widehat{K} \in \mathscr{P}\left(\widehat{D}^{2}, V\right)_{i}$.

Proof. (i) For each $s \in S$ let $\sigma_{s} \in \operatorname{Hol}(D, D)$ given by $\sigma_{s}(m):=s . m$. Then $\sigma_{s}$ induces a continuous algebra homomorphism $\widetilde{\sigma}_{s}: S(\operatorname{Hol}(D)) \rightarrow S(\operatorname{Hol}(D))$ by $\widetilde{\sigma}_{s}(\varphi)(f)=\varphi\left(f \circ \sigma_{s}\right)$. This shows in particular that $\sigma_{s}$ lifts to a holomorphic map $\sigma_{s}^{\prime}: \widehat{D} \rightarrow \widehat{D}$. The action of $S$ on $\widehat{D}$ is now given by $S \times \widehat{D} \rightarrow \widehat{D},(s, m) \mapsto \sigma_{s}^{\prime} . m$.

(ii) As Hol $(D)$ and Hol $(\widehat{D})$ are Fréchet spaces, the Open Mapping Theorem implies that the restriction mapping $r$ : $\mathrm{Hol}(\widehat{D}) \rightarrow \mathrm{Hol}(D)$ is an isomorphism. In view of $\operatorname{Hol}(D, V) \cong \mathrm{Hol}(D) \widehat{\otimes}_{\varepsilon} V$ and $\operatorname{Hol}(\widehat{D}, V) \cong \mathrm{Hol}(\widehat{D}) \widehat{\otimes}_{\varepsilon} V$ (cf. Example I.5), the same holds for the restriction mapping $\operatorname{Hol}(\widehat{D}, V) \rightarrow \operatorname{Hol}(D, V)$ (cf. $\left[\right.$ Tr67, Prop.43.7]). Thus we obtain a realization of $\mathscr{H}_{K}$ in $\operatorname{Hol}(\widehat{D}, V)$, i.e., $K$ extends to a holomorphic positive definite $B(V)$-valued kernel $\widehat{K}$ on $\widehat{D}$. Using the Identity Theorem for Holomorphic Functions, we see that $\widehat{K}$ is $S$-invariant, i.e., $\widehat{K} \in \mathscr{P}\left(\widehat{D}^{2}, V\right)_{i}$ proving the assertion.

\section{Biinvariant Domains in Complex Ol'shanskir Semigroups}

In this chapter we specialize to an important class of complex manifolds, namely biinvariant domains in complex Ol'shanskir semigroups.

Definition $\mathbb{I I I}$.1. Let $g$ be a finite dimentional Lie algebra over $\mathbb{R}$.

(a) An element $X \in \mathrm{g}$ is called elliptic if ad $X$ operates semisimply with purely imaginary spectrum. A convex cone $W \subseteq \mathfrak{g}$ is said to be elliptic if $W^{0} \neq \emptyset$ and all $X \in W^{0}$ are elliptic.

(b) For a subalgebra $a \subseteq g$ we write $\operatorname{Inn}(\mathfrak{a}):=\left\langle e^{\text {ad } a}\right\rangle \subseteq$ Aut $(\mathfrak{g})$ for the corresponding group of inner automorphisms. A subalgebra $\mathfrak{a} \subseteq \mathfrak{g}$ is said to be compactly embedded if $\operatorname{Inn}(\mathfrak{a})$ is relatively compact in Aut $(\mathfrak{g})$.

Remark III.2. (a) If a Lie algebra $g$ admits an elliptic cone $W$, then there exists a compactly embedded Cartan subalgebra $\mathrm{t} \subseteq \mathfrak{g}$ (cf. [Ne99, Th. VII.1.8]).

(b) Suppose that $\mathfrak{g}$ admits a compactly embedded Cartan subalgebra $\mathbf{t}$ and let $E$ $\subseteq \mathrm{g}$ be a Inn $(\mathrm{g})$-invariant subset consisting of elliptic elements. Then $E$ can be reconstructed from its trace in $\mathrm{t}$, i.e., $E=\operatorname{Inn}(\mathfrak{g})$. $(E \cap \mathrm{t})$. This follows for instance from the fact that each elliptic element is contained in a compactly embedded Cartan subalgebra and the fact that all compactly Cartan subalgebras of $g$ are conjugate under Inn $(\mathrm{g})$ (cf. [Ne.99, Th. VII.1.4]). 
From now on we assume that $\mathfrak{g}$ contains a compactly embedded Cartan subalgebra $t$ and that there exists an elliptic cone $W \subseteq \mathfrak{g}$.

Definition III.3. (a) Let $W \subseteq \mathfrak{g}$ be a closed elliptic cone. Let $\widetilde{G}$, resp. $\widehat{G} \mathbb{C}$, be the simply connected Lie groups associated to $\mathfrak{g}$, resp. $\mathfrak{g}_{\mathbb{C}}$, and set $G_{1}:=\langle\exp \mathfrak{g}\rangle$ $\subseteq \widehat{G} \mathbb{c}$. Then Lawson's Theorem (cf. [HiNe93, Th. 7.34, 35]) says that the subset $\Gamma_{G_{1}}(W):=G_{1} \exp (i W)$ is a closed subsemigroup of $G \mathbb{C}$ and the polar map

$$
G_{1} \times W \rightarrow \Gamma_{G_{1}}(W), \quad(g, X) \mapsto g \exp (i X)
$$

is a homeomorphism.

Now the universal covering semigroup $\Gamma_{\widetilde{G}}(W):=\widetilde{\Gamma}_{G_{1}}(W)$ has a similar structure. We can lift the exponential function exp: $g+i W \rightarrow \Gamma_{G_{1}}(W)$ to an exponential mapping Exp: $\mathfrak{g}+i W \rightarrow \Gamma_{\widetilde{G}}(W)$ with $\operatorname{Exp}(0)=\mathbf{1}$ and thus obtain a polar map

$$
\widetilde{G} \times W \rightarrow \Gamma_{\widetilde{G}}(W), \quad(g, X) \mapsto g \operatorname{Exp}(i X)
$$

which is a homeomorphism.

If $G$ is a connected Lie group associated to $g$, then $\pi_{1}(G)$ is a discrete central subgroup of $\Gamma_{\widetilde{G}}(W)$ and we obtain a covering homomorphism $\Gamma_{\widetilde{G}}(W) \rightarrow$ $\Gamma_{G}(W):=\Gamma_{\widetilde{G}}(W) / \pi_{1}(G)$ (cf. [HiNe93, Ch. 3]). It is easy to see that there is also a polar map $G \times W \rightarrow \Gamma_{G}(W),(g, X) \mapsto g \operatorname{Exp}(i X)$ which is a homeomorphism. The semigroups of the type $\Gamma_{G}(W)$ are called complex Ol'shanskiı semigroups.

The subset $\Gamma_{G}\left(W^{0}\right) \subseteq \Gamma_{G}(W)$ is an open semigroup carrying a complex manifold structure such that semigroup multiplication is holomorphic. Moreover there is an involution on $\Gamma_{G}(W)$ given by

$$
*: \Gamma_{G}(W) \rightarrow \Gamma_{G}(W), s=g \operatorname{Exp}(i X) \mapsto s^{*}=\operatorname{Exp}(i X) g^{-1}
$$

which is antiholomorphic on $\Gamma_{G}\left(W^{0}\right)$ (cf. [HiNe93,Th. 9.15] for a proof of all. that). Thus $\Gamma_{G}(W)$ is an involutive semigroup.

(b) A biinvariant domain $D \subseteq \Gamma_{G}\left(W^{0}\right)$ is an open connected $G \times G$ biinvariant subset of $\Gamma_{G}\left(W^{0}\right)$. Note that

$$
D=G \operatorname{Exp}\left(D_{h}\right)=G \operatorname{Exp}(\mathscr{D}) G,
$$

where $D_{h} \subseteq i W^{0}$ and $\mathscr{D}=D_{h} \cap i$ (cf. Remark III.2(b)).

Theorem III.4. (K.-H. Neeb) All complex Ol'shanski are Stein manifolds. Further if $D \subseteq \Gamma_{G}\left(W^{0}\right)$ is a biinvariant domain, then its envelope of holomorphy (cf. Definition II.13) $\widehat{D}$ of $D$ is given by

$$
\widehat{D}=G \operatorname{Exp}\left(\operatorname{conv}\left(D_{h}\right)\right) .
$$

Proof. [Ne98, Th. 5.18, Th. 7.9]. 
Definition III.5. A Hilbert space $\mathscr{H}$ which is a subspace of Hol $(D)$ is called a biinvariant Hilbert space if the following conditions are satisfied: (BH1) The inclusion $\mathscr{H} C \operatorname{Hol}(D)$ is continuous. (BH2) The group $G \times G$ acts unitarily on $\mathscr{H}$ via

$$
\left(\left(g_{1}, g_{2}\right) . f\right)(z)=f\left(g_{1}^{-1} z g_{2}\right)
$$

for $g_{1}, g_{2} \in G, z \in D$.

Lemma III.6. Let $G \times G$ act on $D$ by $\left(g_{1}, g_{2}\right) . z=g_{1} z g_{2}^{-1}$ and consider $G$ $\times G$ as an involutive semigroup with involution $\left(g_{1}, g_{2}\right) *:=\left(g_{1}^{-1}, g_{2}^{-1}\right)$. Write $\mathscr{P}\left(D^{2}\right)_{i}$ for the $G \times G$-invariant holomorphic positive definite kernels.

(i) The prescription $K \mapsto\left(\pi_{K}, \mathscr{H}_{K}\right)$ defines a surjective correspondence between $\mathscr{P}\left(D^{2}\right)_{i}$ and the set of biinvariant Hilbert spaces.

(ii) For every $K \in \mathscr{P}\left(D^{2}\right)_{i}$, the corresponding representation $\left(\pi_{K}, \mathscr{H}_{K}\right)$ of $G \times G$ is multiplicity-free.

Proof. (1) Let $K \in \mathscr{P}\left(D^{2}\right)_{i}$. Then [Ne99, Th. II.4.4] together with the invariance of $K$ implies that $\left\|\pi_{K}^{0}\left(g_{1}, g_{2}\right)\right\|=1$ for all $\left(g_{1}, g_{2}\right) \in G \times G$ and thus $\left(\pi_{K}^{0}, \mathscr{H}_{K}^{0}\right)$ extends to an involutive representation of $\left(\pi_{K}, \mathscr{H}_{K}\right)$ of $G \times G$. Since $\pi_{K}\left(\left(g_{1}, g_{2}\right)^{-1}\right)=\pi_{K}\left(\left(g_{1}, g_{2}\right)^{*}\right)=\pi_{K}\left(g_{1}, g_{2}\right)^{*}$, this representation is by unitary operators. Finally the continuity of the action of $G \times G$ on $D$ implies that $\left(\pi_{K}\right.$, $\left.\mathscr{H}_{K}\right)$ is weakly continuous, hence unitary. Thus $K \mapsto\left(\pi_{K}, \mathscr{H}_{K}\right)$ is well defined.

It remains to show that the correspondence is onto. Let $\mathscr{H}$ be a biinvariant Hilbert space. This means in particular that all point evaluations $\mathscr{H} \rightarrow \mathbb{C}, f \mapsto$ $f(z)$ are continuous for $z \in D$. Then $f(z)=\left\langle f, K_{z}\right\rangle$ for some $K_{z} \in \mathscr{H}$ and $\mathscr{H}=\mathscr{H}_{K}$, where $K(z, w)=\left\langle K_{w}, K_{z}\right\rangle$ for $z, w \in D$. Moreover the invariance of $\mathscr{H}$ under $G \times$ $G$ implies that $K\left(g_{1} z g_{2}^{-1}, w\right)=K\left(z, g_{1}^{-1} w g_{2}\right)$ for all $z, w \in D, g_{1}, g_{2} \in G$, i.e., $K \in$ $\mathscr{P}\left(D^{2}\right)_{i}$.

(ii) [Ne97, Ex. II.4].

In view of Proposition II.14 and Theorem III.4, it follows from Lemma III.6(i) that the discussion of biinvariant Hilbert spaces $\mathscr{H} \subseteq \mathrm{Hol}(D)$ is reduced to the case where $D_{h}$ is convex. From now on we will make this assumption.

Proposition III.7. Let $\mathscr{H}_{K} \subseteq \operatorname{Hol}(D)$ be a biinvariant Hilbert space and let $\gamma: T \rightarrow \operatorname{Ext}\left(\mathscr{P}\left(D^{2}\right)_{i}\right), t \mapsto K^{t}$ be an admissible parametrization. (cf. Definition I.6 (e)). Then there exists a unique Radon measure $\mu$ on $T$ such that

$$
K=\int_{T} K^{t} d \mu(t)
$$

and a unitary equivalence 


$$
\left(\pi_{K}, \mathscr{H}_{K}\right) \cong\left(\int_{T}^{\oplus} \pi_{t} d \mu(t), \int_{T}^{\oplus} \mathscr{H}_{t} d \mu(t)\right)
$$

of $G \times G$-modules.

Proof. In view of Lemma III.6(ii), this is a direct consequence of Theorem II. 12 .

\section{The Plancherel Theorem for Biinvariant Hilbert Spaces}

In this chapter we finally derive the Plancherel Theorem. In view of Proposition III.7, the main task hereby is to clarify which kind of representations $\left(\pi_{t}, \mathscr{H}_{t}\right)$ occur in the integral decomposition of $\left(\pi_{K}, \mathscr{H}_{K}\right)$ and what the parameter space $T$ could be. It turns out that the occuring representations $\left(\pi_{t}, \mathscr{H}_{t}\right)$ in the integral decomposition of $\left(\pi_{K}, \mathscr{H}_{K}\right)$ are highest weight representations and that there is an admissible parametrization of $\operatorname{Ext}\left(\mathscr{P}\left(D^{2}\right)_{\imath}\right)$ by highest weights.

\section{Highest Weight Representations}

To step further we first need some terminology concerning Lie algebras with compactly embedded Cartan subalgebras.

Definition IV.1. Let $\mathfrak{g}$ be a real algebra admitting a compactly embedded Cartan subalgebra t.

(a) Associated to the Cartan subalgebra $t_{\mathbb{C}}$ in the complexification $g_{\mathbb{C}}$ is a root decomposition as follows. For a linear functional $\alpha \in \mathfrak{t}_{\mathbb{C}}^{*}$ we set

$$
\mathfrak{g}_{\mathbb{C}}^{\alpha}:=\left\{X \in \mathfrak{g}_{\mathbb{C}}:\left(\forall Y \in \mathfrak{t}_{\mathbb{C}}\right)[Y, X]=\alpha(Y) X\right\}
$$

and write $\Delta:=\left\{\alpha \in \mathrm{t}_{\mathbb{C}}^{*} \backslash\{0\}: \mathrm{g}_{\mathbb{C}}^{\alpha} \neq\{0\}\right\}$ for the set of roots. Then $\mathrm{g}_{\mathbb{C}}=\mathrm{t}_{\mathbb{C}} \oplus \oplus_{\alpha \in \Delta} \mathrm{g}_{\mathbb{C}}^{\alpha}$, $\alpha(\mathrm{t}) \in i \mathbb{R}$ for all $\alpha \in \Delta$ and $\overline{\mathrm{g}_{\mathbb{C}}^{\alpha}}=\mathrm{g}_{\mathbb{C}} \overline{\mathbb{C}}^{\alpha}$, where $X \rightarrow \bar{X}$ denotes complex conjugation on $\mathfrak{g}_{\mathbb{C}}$ with respect to $\mathfrak{g}$.

(b) Let be a maximal compactly embedded subalgebra of $g$ containing $\mathfrak{t}$. Note that $\mathfrak{k}$ is unique (cf. [KrNe96, Cor. III.8]). A root $\alpha$ is said to be compact if $\mathfrak{g}_{\mathbb{C}}^{\alpha}$ $\subseteq \mathfrak{f}_{\mathbb{C}}$ and non-compact otherwise. We write $\Delta_{k}$ for the set of compact roots and $\Delta_{n}$ for the non-compact ones. If $\mathfrak{g}=\mathfrak{r} \times \mathfrak{g}$ is a $\mathfrak{l}$-invariant Levi decomposition, then we set

$$
\Delta_{r}:=\left\{\alpha \in \Delta: g_{\mathbb{C}}^{\alpha} \in \mathfrak{r}_{\mathbb{C}}\right\} \text { and } \Delta_{s}:=\left\{\alpha \in \Delta: \mathfrak{g}_{\mathbb{C}}^{\alpha} \in \mathfrak{g}_{\mathbb{C}}\right\}
$$

and recall that $\Delta=\Delta_{r} \dot{U} \Delta_{s}$ (cf. [Ne99, Ch. VII]). Further we write $\Delta_{n, s}:=\Delta_{n} \cap \Delta_{s}$ for the set of all non-compact semisimple roots.

(c) A positive system $\Delta^{+}$of roots is a subset of $\Delta$ for which there exists a 
regular element $X_{0} \in i$ t $^{*}$ with $\Delta^{+}:=\left\{\alpha \in \Delta: \alpha\left(X_{0}\right)>0\right\}$. A positive system is said to be $\mathfrak{l}^{-a d a p t e d}$ if the set $\Delta_{n}^{+}:=\Delta_{n} \cap \Delta^{+}$is invariant under the Weyl group $\mathscr{W}_{\mathrm{t}}:=N_{\text {Inn(t) }}(\mathrm{t}) / Z_{\text {Inn(t) }}(\mathrm{t})$ acting on $\mathrm{t}$. We recall from $[\mathrm{Ne} 99, \mathrm{Ch}$. VII] that there exists a $\mathfrak{l}$-adapted positive system if and only if $z g(z(\mathfrak{z}))=\mathfrak{l}$. In this case we call $g$ quasihermitian. In this case it is easy to see that $\mathscr{B}$ is quasihermitian too, and so all simple ideals of $\mathfrak{B}$ are either compact or hermitian.

(d) We associate to a positive system $\Delta^{+}$the convex cones

$$
\begin{gathered}
C_{\text {min }}:=\operatorname{cone}\left\{i\left[\overline{X_{\alpha}}, X_{\alpha}\right]: X_{\alpha} \in g_{\mathbb{C}}^{\alpha}, \alpha \in \Delta_{n}^{+}\right\}, \\
C_{\text {min }, z}:=C_{\min } \cap z(\mathfrak{g})=\operatorname{cone}\left\{i\left[\overline{X_{\alpha}}, X_{\alpha}\right]: X_{\alpha} \in g_{\mathbb{C}}^{\alpha}, \alpha \in \Delta_{r}^{+}\right\}
\end{gathered}
$$

and $C_{\max }:=\left(i \Delta_{n}^{+}\right)=\left\{X \in \mathrm{t}:\left(\forall_{\alpha} \in \Delta_{n}^{+}\right) i \alpha(X) \geq 0\right\}$. Note that both $C_{\min }$ and $C_{\text {max }}$ are closed convex cones in $\mathrm{t}$.

(e) Write $p_{t}: \mathrm{g} \rightarrow \mathrm{t}$ for the orthogonal projection along $[\mathrm{t}, \mathrm{g}]$ and set $\mathscr{O}_{X}:=$ Inn(g). $X$ for the adjoint orbit through $X \in \mathfrak{g}$. We define the maximal cone associated to $\Delta^{+}$by

$$
W_{\max }:=\left\{X \in \mathrm{g}: p_{t}\left(\mathscr{O}_{X}\right) \subseteq C_{\max }\right\}
$$

and note that $W_{\max }$ is a closed convex $\operatorname{Inn}(\mathrm{g})$-invariant cone in $\mathrm{g}$.

Definition IV.2. Let $\Delta^{+}$be a positive system.

(a) For a $\mathfrak{g}_{\mathbb{C}^{-m o d u l e}} V$ and $\beta \in\left(\mathrm{t}_{\mathbb{C}}\right)^{*}$ we write $V^{\beta}:=\left\{v \in V:\left(\forall X \in \mathrm{t}_{\mathbb{C}}\right) X . v=\right.$ $\beta(X) v\}$ for the weight space of weight $\beta$ and $\mathscr{P}_{V}=\left\{\beta: V^{\beta} \neq\{0\}\right\}$ for the set of weights of $V$.

(b) Let $V$ be a $\mathfrak{g}_{\mathbb{C}^{-}}$module and $v \in V^{\lambda}$ a $\mathbb{t}_{\mathbb{C}^{-}}$weight vector. We say that $v$ is a primitive element of $V$ (with respect to $\Delta^{+}$) if $\mathfrak{g}_{\mathbb{C}}^{\alpha} \cdot v=\{0\}$ holds for all $\alpha \in \Delta^{+}$.

(c) $\mathrm{A} \mathfrak{g}_{\mathbb{C}^{-}}$-module $V$ is called a highest weight module with highest weight $\lambda$ (with respect to $\Delta^{+}$) if it is generated by a primitive element of weight $\lambda$.

(d) Let $\lambda \in i$ t $^{*}$ be dominant integral with respect to $\Delta_{k}^{+}$and $F(\lambda)$ the corresponding highest weight module for $\mathfrak{l}_{\mathbb{C}}$. Assume that $\Delta^{+}$is $\mathfrak{E}$-adapted and set $\mathfrak{p}^{ \pm}=\bigoplus_{\alpha \in \Delta_{n}^{ \pm}} \mathrm{g}_{\mathbb{C}}^{\alpha}$. We define the generalized Verma module by

$$
N(\lambda):=u\left(\mathfrak{g}_{\mathbb{C}}\right) \otimes_{u_{\left(\mathbb{E} \mathbb{C}+\mathfrak{p}^{+}\right.} F} F(\lambda) .
$$

Note that $N(\lambda)$ is a highest weight module for $\mathcal{U}\left(g_{\mathbb{C}}\right)$ with highest weight $\lambda$. We denote by $L(\lambda)$ the unique irreducible quotient of $N(\lambda)$.

(e) Let $G$ be a connected Lie group with Lie algebra g. We write $K$ for the analytic subgroup of $G$ corresponding to $\mathscr{L}$. Let $(\pi, \mathscr{H})$ be a unitary representation of $G$. A vector $v \in \mathscr{H}$ is called $K$-finite if it is contained in a finite dimensional $K$-invariant subspace. We write $\mathscr{H}^{K, \omega}$ for the space of analytic $K$-finite vectors.

(f) An irreducible unitary representation $(\pi, \mathscr{H})$ of $G$ is called highest weight 
representation with respect to $\Delta^{+}$with highest weight $\lambda \in i \mathrm{t}^{*}$ if $\mathscr{H}^{K, \omega}$ is a highest weight module for $\mathfrak{g}_{\mathbb{C}}$ with respect to $\Delta^{+}$and highest weight $\lambda$. We say that the irreducible highest weight module $L(\lambda)$ is unitarizable if there exists a unitary highest weight representation $\left(\pi_{\lambda}, \mathscr{H}_{\lambda}\right)$ of $\widetilde{G}$ with $\mathscr{H}_{\lambda}^{K, \omega} \cong L(\lambda)$ as $g_{\mathrm{c}}$-modules. We write $H W\left(G, \Delta^{+}\right) \subset i t^{*}$ for the set of highest weight corresponding to highest weight representations of $G$ with respect to $\Delta^{+}$and set $H W\left(\Delta^{+}\right):=H W\left(\widetilde{G}, \Delta^{+}\right)$ for the set of all unitarizable highest weights with respect to $\Delta^{+}$.

(g) Let $\lambda \in H W\left(\Delta^{+}\right)$. We call $\lambda$ singular if the natural map $N(\lambda) \rightarrow L(\lambda)$ has a non-trivial kernel and non-singular otherwise.

Let $W \subseteq \mathrm{g}$ be a closed elliptic cone. Recall from [KrNe96, Th. IV .6] that there exists a ${ }^{-}$-adapted positive system $\Delta^{+}$such that

$$
C_{\min } \subseteq W \cap \mathrm{t} \subset C_{\max }
$$

holds. From now on we fix a positive system $\Delta^{+}$having this property. Then $W_{\max }$ is an elliptic cone and we have $C_{\max }=W_{\max } \cap \mathrm{t}$ (cf. [KrNe96, Cor. IX.10]).

For each unitary representation $(\pi, \mathscr{H})$ of $G$ we write $\left(\pi^{*}, \mathscr{H}^{*}\right)$ for the corresponding dual representation. Let $B_{2}(\mathscr{H})$ be the space of Hilbert Schmidt operators on $\mathscr{H}$. We define a representation of $G \times G$ on $B_{2}(\mathscr{H})$ by

$$
\pi^{c}: G \times G \rightarrow U\left(B_{2}(\mathscr{H})\right), \pi^{\mathrm{c}}\left(g_{1}, g_{2}\right) . A:=\pi\left(g_{2}\right) A \pi\left(g_{1}\right)^{*} .
$$

Note that there is canonical isomorphism between $\left(\pi^{*} \otimes \pi, \mathscr{H}^{*} \widehat{\otimes} \mathscr{H}\right)$ and $\left(\pi^{c}\right.$, $\left.B_{2}(\mathscr{H})\right)$. We write $\|\cdot\|_{2}$ for the Hilbert-Schmidt norm on $B_{2}(\mathscr{H})$ and $\|\cdot\|_{1}$ for the norm on the trace class operators $B_{1}(\mathscr{H})$.

Recall from [Ne99, Th. XI.4.5] that each highest weight representation $\left(\pi_{\lambda}, \mathscr{H}_{\lambda}\right)$ of $G$ extends to an holomorphic representation of $\Gamma_{\mathrm{G}}\left(W_{\max }\right)$ denoted by the same symbol. Moreover all operators $\pi_{\lambda}(s), s \in \Gamma_{G}\left(W_{\max }^{0}\right)$, are of trace class (cf. [Ne94, Th. III.8]), so that the notion $\Theta_{\lambda}(s):=\operatorname{tr} \pi_{\lambda}(s)$ makes sense for all $s \in \Gamma_{G}\left(W_{\max }^{0}\right)$. We call $\Theta_{\lambda}$ the character of $\left(\pi_{\lambda}, \mathscr{H}_{\lambda}\right)$ and note that $\Theta_{\lambda}$ is holomorphic on $\Gamma_{G}\left(W_{\max }^{0}\right)$ (cf. [Ne94, Th. IV.11]).

Proposition IV.3. Let $D \subseteq \Gamma_{G}\left(W_{\max }^{0}\right)$ be a biinvariant domain and $K \in$ $\operatorname{Ext}\left(\mathscr{P}\left(D^{2}\right)_{i}\right)$.

(i) There exists a unitary highest weight representation $\left(\pi_{\lambda}, \mathscr{H}_{\lambda}\right)$ of $G$ and a constant $c>0$ such that the mapping

$$
\left(\pi_{\lambda}^{c}, B_{2}\left(\mathscr{H}_{\lambda}\right)\right) \rightarrow\left(\pi_{K}, \mathscr{H}_{K}\right), \quad A \mapsto\left(s \mapsto c \operatorname{tr}\left(A \pi_{\lambda}(s)\right)\right)
$$

is an unitary equivalence of $G \times G$-modules.

(ii) The face $F_{K}$ is uniquely determined by $\lambda$. 
Proof. (i) [Ne99, Th. XIII.8.11].

(ii) Suppose the assertion is false. Then there exists $K_{1}, K_{2} \in \operatorname{Ext}\left(\mathscr{P}\left(D^{2}\right)_{i}\right)$ with $F_{K_{1}} \neq F_{K_{2}}$, but $\left(\pi_{K_{1}}, \mathscr{H}_{K_{1}}\right) \cong\left(\pi_{\lambda}^{c}, B_{2}\left(\mathscr{H}_{\lambda}\right)\right) \cong\left(\pi_{K_{2}}, \mathscr{H}_{K_{2}}\right)$. Set $L:=K_{1}+K_{2}$. Then $L$ $\in \mathscr{P}\left(D^{2}\right)_{i}$ and $\mathscr{H}_{L}=\mathscr{H}_{K_{1}}+\mathscr{H}_{K_{2}}$ (cf. [Ne99, Rem. I. 12]). Moreover, [Ne99, Th. I. 16] shows that $\mathscr{H}_{K_{1}} \neq \mathscr{H}_{K_{2}}$ and thus $\mathscr{H}_{K_{1}} \cap \mathscr{H}_{K_{2}}=\{0\}$ by the irreducibility of the representations $\left(\pi_{K_{1}}, \mathscr{H}_{K_{1}}\right)$ and $\left(\pi_{K_{2}}, \mathscr{H}_{K_{2}}\right)$. Now [Ne99, Rem. I. 12] implies that $\mathscr{H}_{K_{1}} \oplus \mathscr{H}_{K_{2}}=\mathscr{H}_{L}$ is an orthogonal direct sum. Thus

$$
\left(\pi_{L}, \mathscr{H}_{L}\right) \cong\left(\pi_{\lambda}^{c} \oplus \pi_{\lambda}^{c}, B_{2}\left(\mathscr{H}_{\lambda}\right) \oplus B_{2}\left(\mathscr{H}_{\lambda}\right)\right),
$$

i.e., $\left(\pi_{L}, \mathscr{H}_{L}\right)$ is not multiplicity-free, contradicting Lemma III.6(ii).

In the sequel we realize $\left(\pi_{\lambda}^{c}, B_{2}\left(\mathscr{H}_{\lambda}\right)\right)$ in $\operatorname{Hol}\left(\Gamma_{G}\left(W_{\max }^{0}\right)\right)$ via the map in Proposition IV.3(i) for $D=\Gamma_{G}\left(W_{\max }^{0}\right)$ and $c=1$. Note that the corresponding reproducing kernel is given by $K^{\lambda}(s, t):=\Theta_{\lambda}\left(s t^{*}\right)$ for all $s, t \in \Gamma_{G}\left(W_{\max }^{0}\right)$ (cf. [Ne94, Th. IV.11]).

We also write $K^{\lambda}:=\left.K^{\lambda}\right|_{D \times D}$ for the restriction of $K^{\lambda}$ to $D \times D$. Proposition IV. 3 implies in particular that the map

$$
\gamma: H W\left(G, \Delta^{+}\right) \rightarrow \operatorname{Ext}\left(\mathscr{P}\left(D^{2}\right)_{i}\right), \lambda \mapsto K^{\lambda}
$$

is injective and that $\operatorname{im} \gamma$ is a section of $\operatorname{Ext}\left(\mathscr{P}\left(D^{2}\right)_{i}\right)$. In the remaining part of this section we will be concerned with the prove that $\gamma$ is in fact an admissible parametrization. From that the Plancherel Theorem will follow.

Remark IV.4. We call $\mathfrak{g}$ admissible if the direct sum $\mathfrak{g} \oplus \mathbb{R}$ admits pointed invariant elliptic cones. If $D$ is a biinvariant domain associated to $\mathfrak{g}$, then there exists an admissible quotient $g_{1}$ of $\mathfrak{g}$, a biinvariant domain $D_{1}$ associated to $g_{1}$ and an equivariant holomorphic quotient map $q: D \rightarrow D_{1}$. Further every $K \in$ $\mathscr{P}\left(D^{2}\right)_{i}$ factors to a holomorphic positive definite biinvariant kernel $K_{1}$ on $D_{1}$ via $K_{1}(q(z), q(w)):=K(z, w)$ for all $z, w \in D$. In particular, we have $\mathscr{H}_{K} \cong \mathscr{H}_{K_{1}}$, and thus $\mathscr{H}_{K}$ can be realized as a biinvariant Hilbert space in $\mathrm{Hol}\left(D_{1}\right)$ (cf. [Ne99, Ch. XIII] for all that). For what this restriction procedure means in the more concrete setting of Hardy and Bergman spaces we refer to [Kr98a, Rem. III.6, Lemma II.9].

Our objective is to obtain an integral representation for kernels $K \in$ $\mathscr{P}\left(D^{2}\right)_{i}$. To achieve this, Remark IV.4 tells us that it is no restriction to assume from the beginning that $\mathfrak{g}$ is admissible. From now on we will make this assumption.

\section{The Set of Highest Weights}

This subsection is devoted to an explicit description of the set of all 
unitary highest weights $H W\left(\Delta^{+}\right) \subseteq i t^{*}$. For subsets $A, B \subseteq i t^{*}$ we define their minimal distance by

$$
\delta_{\min }(A, B):=\inf _{x \in A, y \in B}\|x-y\|,
$$

where $\|\cdot\|$ denotes a norm $i$ t*.

Lemma IV.5. (1) If $\mathfrak{g}$ is simple hermitian and $\zeta \in i z(\mathfrak{k}) *$ such that $\zeta(\breve{\beta})=$ 1 , where $\beta$ is the maximal root in $\Delta^{+}$, then

$$
H W\left(\Delta^{+}\right)=D \cup \bigcup_{m \in \mathbb{N}} R_{m},
$$

where $D$ is a countable discrete closed subset of it and $R_{m}=\lambda_{m}-\mathbb{R}^{+} \zeta, m \in \mathbb{N}$, is a ray. The singular elements $H W\left(\Delta^{+}\right)$are given by $D \cup\left\{\lambda_{m}: m \in \mathbb{N}\right\}$. Moreover, there exists a constant $C>0$ such that $\delta_{\min }\left(D, R_{n}\right), \delta_{\min }\left(R_{n}, R_{m}\right)>C$ holds for all $m, n \in$ $\mathbb{N}, m \neq n$.

(ii) If $\mathrm{g}$ is a compact semisimple, then

$$
H W\left(\Delta^{+}\right)=\left\{\lambda \in i \mathrm{t}^{*}:\left(\forall \lambda \in \Delta^{+}\right) \frac{2\langle\lambda, \alpha\rangle}{\langle\alpha, \alpha\rangle} \in \mathbb{N}_{0}\right\}
$$

In particular, $H W\left(\Delta^{+}\right)$is a countable discrete closed subset of it*.

Proof. (i) This follows from [EHW83, Prop. 3.1, Cor. 3.16].

(ii) This is part of the Theorem of Highest Weight for reductive Lie algebras.

Let $\mathfrak{g}=\mathfrak{r} \times \mathfrak{g}$ be a $\mathfrak{f}$-invariant Levi decomposition. Let $\mathfrak{u} \subseteq \mathfrak{g}$ be the nilradical and $\mathrm{t}_{0}$ be a complement to $\mathfrak{z}(\mathfrak{g})$ in $\mathfrak{t} \cap \mathfrak{r}$. Then $\mathfrak{l}:=\mathrm{t}_{0} \oplus \mathfrak{g}$ is a reductive subalgebra of $\mathfrak{g}$ and $\mathfrak{g}=\mathfrak{u} \times \mathfrak{I}$ is a $\mathfrak{L}$-invariant semidirect decomposition. Let $\widetilde{G}=$ $\widetilde{U} \rtimes \widetilde{L}$ be the associated semidirect product on the level of simply connected groups. Further we set $\mathrm{t}_{\mathfrak{l}}:=\mathrm{t} \cap \mathfrak{l}$ and note that $\mathrm{t}=\mathfrak{z}_{\mathrm{z}}(\mathfrak{g}) \oplus \mathrm{t}_{\mathrm{l}}$.

Let $\mathfrak{g}=\oplus_{1=1}^{n} \mathfrak{g}_{j}$ be the decomposition in simple ideals with $\mathfrak{g}_{j}, 1 \leq j \leq r$, compact and $\mathfrak{S}_{j}, r+1 \leq j \leq n$, hermitian (cf. Definition IV.1 (e)). Associated to the direct sum decomposition $\mathfrak{l}=\mathrm{t}_{0} \oplus \bigoplus_{j=1}^{n} \mathfrak{G}_{j}$ there is a splitting $\widetilde{L}=\widetilde{T_{0}} \times \prod_{j=1} \widetilde{S_{j}}$ of $\tilde{L}$ into simply connected factors. For each $1 \leq j \leq n$ we set $\mathrm{t}_{j}:=\mathrm{t} \cap \mathfrak{g}_{j}$.

Lemma IV.6. If $\mathrm{g}=\mathfrak{l}$ is reductive, then

$$
H W\left(\Delta^{+}\right)=\bigcup_{m \in \mathbb{N} I \subseteq\{r+1, \ldots, n\}} C_{m, I}
$$

is a countable disjoint union of convex affine cones. Moreover:

(i) The occuring cones $C_{m, I}$ have the form

$$
C_{m, I}=i \mathrm{t}_{0}^{*}+\sum_{j=1}^{r} \lambda_{j}^{m}+\sum_{j \in\{r+1, \ldots, n\} \backslash I} \lambda_{j}^{m}+\sum_{j \in I}\left(\lambda_{j}^{m}-\right] 0, \infty\left[\zeta_{j}\right),
$$


where $\lambda_{j}^{m} \in i \mathrm{t}_{j}^{*}$ for all $1 \leq j \leq n$. Further, all $\lambda_{j}^{m}, j \in\{r+1, \ldots, n\} \backslash I$, are singular for $\tilde{S_{j}}$, and $\left.\lambda_{j}^{m}-\right] 0, \infty\left[\zeta_{j}, j \in I\right.$, consists of non-singular elements for $\tilde{S_{j}}$.

(ii) The closure of $C_{m, I}$ is given by

$$
\overline{C_{m, I}}=\bigcup_{J \subseteq I} C_{m, J} .
$$

(iii) Set $C_{m}:=\cup_{I \subseteq\{r+1, \ldots, n\}} C_{m, I}$. Then there exists a constant $C>0$ such that the minimal distances satisfy $\delta_{\min }\left(C_{m}, C_{n}\right)>C$ for all $m, n \in \mathbb{N}, m \neq n$.

Proof. This is a direct consequence of Lemma IV.5.

.

Note that a necessary condition for $L(\lambda)$ to be unitarizable is $\lambda \in i C_{\text {min }}^{\text {in }}$ (cf. [Ne96, Lemma I.4 (iii)]). Thus $H W\left(\Delta^{+}\right) \subseteq i C_{\text {min }}^{\star}$ and $\left.H W\left(\Delta^{+}\right)\right|_{i z(g)} \subseteq i C_{\text {min, } z \text {. Let }}$ $\mathfrak{m}^{+}:=\bigoplus_{\alpha \in \Delta_{r}^{+}} g_{\mathbb{C}}^{\alpha}$ and note that $\left[\mathrm{m}^{+}, \overline{\mathrm{m}^{+}}\right] \subseteq \mathfrak{z}\left(\mathfrak{g}_{\mathbb{C}}\right)$ as $g$ was assumed to be admissible (cf. [HiNe93, Th. 7.15]).

Each $\mu \in i C_{\mathrm{m}}^{\mathrm{m}, z}$ induces a positive semidefinite hermitian form on $\mathrm{m}^{+}$by

$$
\psi_{\mu}: \mathfrak{m}^{+} \times \mathfrak{m}^{+} \rightarrow \mathbb{C}, \quad(X, Y) \mapsto \mu([\bar{Y}, X])
$$

(cf. [Ne.96, Sect. III]). For each $\alpha \in \Delta_{r}^{+}$, we set

$$
W^{\alpha}(\mu):=\left\{X \in \mathfrak{g}_{\mathbb{C}^{:}}^{\alpha}\left(\forall Y \in \mathfrak{g}_{\mathbb{C}}^{\alpha}\right) \phi_{\mu}(X, Y)=0\right\},
$$

$m_{\alpha}(\mu):=\operatorname{dim}_{\mathbb{C}}\left(\mathfrak{g}_{\mathbb{C}}^{\alpha} / W^{\alpha}(\mu)\right), \rho_{r, \mu}:=\frac{1}{2} \sum_{\alpha \in \Delta_{r}}^{+} m_{\alpha}(\mu) \alpha$ and $\mathfrak{m}_{\mu}^{+}:=\bigoplus_{\alpha \in \Delta_{r}^{+}} \mathfrak{g}_{\mathbb{C}}^{\alpha} / W^{\alpha}(\mu)$.

Note that $\left\{\left(m_{\alpha}(\mu)\right)_{\alpha \in \Delta^{+}} \in \mathbb{N}_{0}^{\Delta^{+}}: \mu \in i C_{\text {min, }}^{\star \star x}\right\}$ is a finite set, say $\left\{f_{1}, \ldots, f_{k}\right\}$. For each $1 \leq j \leq k$ we set $\rho_{r, j}=\frac{1}{2} \sum_{\alpha \in \Delta_{r}^{+}}\left(f_{j}\right)_{\alpha} \alpha$, where $f_{j}=\left(\left(f_{j}\right)_{\alpha}\right)_{\alpha \in \Delta^{t}}$, and associate a not necessarily convex subcone of $i C_{\mathrm{min}, z}$ by

$$
C_{j}:=\left\{\mu \in i C_{\text {min, }}^{\mathrm{i}}:\left(m_{\alpha}(\mu)\right)_{\alpha \in \Delta_{r}^{+}}=f_{j}\right\} .
$$

Note that $i C_{\mathrm{min}, \mathrm{z}}^{\star}=\cup_{j=1}^{k} C_{j}$ is a disjoint union.

For each $\lambda \in i C_{\mathrm{min}}^{\star}$ we set $\lambda_{z}:=\left.\lambda\right|_{i z(g)}$.

Now we have all notation to describe $H W\left(\Delta^{+}\right)$for an arbitrary admissible Lie algebra g. The main tool is the method of metaplectic factorization: Each highest weight representation $\left(\pi_{\lambda}, \mathscr{H}_{\lambda}\right)$ of $\widetilde{G}$ is a tensor product $\left(\nu_{\lambda z} \otimes \pi_{\lambda_{1}}, \mathscr{F}\left(\mathfrak{m}_{\lambda z}^{+}\right)\right.$ $\left.\otimes \pi_{\lambda_{1}}\right)$, where $\left(\nu_{\lambda_{z}}, \mathscr{F}\left(\mathrm{m}_{\lambda_{z}}^{+}\right)\right)$is a so called extended metaplectic representation modelled on the Fock space

$$
\mathscr{F}\left(\mathfrak{m}_{\lambda_{z}}^{+}\right):=\left\{f \in \operatorname{Hol}\left(\mathfrak{m}_{\lambda_{z}}^{+}\right): \int_{m_{\lambda_{z}}^{+}}|f(x)|^{2} e^{-\lambda_{z}([x, \bar{x}])} d \mu_{m_{\lambda_{z}}^{+}}(x)<\infty\right\},
$$

which is a highest weight representation of $\tilde{G}$ with highest weight $\lambda_{z}-\rho_{r, \lambda} \in$ $i(\mathfrak{z}(\mathfrak{E}))^{*}$. Further, $\left(\pi_{\lambda_{1}}, \mathscr{H}_{\lambda_{1}}\right)$ is a highest weight representation of $\widetilde{L}$ with highest 
weight $\lambda_{1}:=\left.\lambda\right|_{i t_{\mathrm{t}}}+\rho_{r, \lambda}$ which is considered to be trivial on $\tilde{U}$ (cf. [Ne96, Sect. III]).

Lemma IV.7. Let $\mathrm{g}$ be an admissible Lie algebra and $\Delta^{+}$be a -adapted positive system. Then:

(i) The set $H W\left(\Delta^{+}\right)$is a countable union of affine cones

$$
H W\left(\Delta^{+}\right)=\bigcup_{m=1}^{\infty} \bigcup_{j=1}^{k} \bigcup_{I \subseteq\{\gamma+1, \ldots, n\}} C_{m, j, I}
$$

where

$$
C_{m, j, I}=-\rho_{r, j}+C_{j}+\left(i C_{\min }^{\star} \cap C_{m, I}\right),
$$

with $C_{m, I} \subseteq i \mathrm{t}_{1}^{*}$ is as in Lemma IV.6 defined with respect to the positive system $\Delta_{s}^{+}$.

(ii) Each $C_{m, j, I}$ is a Borel subset of $i \mathrm{t}^{*}$.

(iii) Let $C_{m}=\cup_{j, I} C_{m, j, I}$. Then there exists a constant $C>0$ such that the minimal distances satisfy $\delta_{\min }\left(C_{m}, C_{n}\right)>C$ for all $m, n \in \mathbb{N}, m \neq n$.

Proof. (1) Let $\lambda \in i C_{\text {min. }}^{\star}$ According to [Ne96, Th. III.9], $L(\lambda)$ is unitarizable if and only if the $\mathbb{I}_{\mathbb{C}}$-module $L\left(\lambda_{1}\right)$, where $\lambda_{1}=\left.\lambda\right|_{i t_{1}}+\rho_{r, \lambda}$ is unitarizable. Thus the assertion follows from Lemma IV.6.

(ii) According to (i) and Lemma IV.6(i), we only have to show that the cones $C_{j}$, $1 \leq i \leq k, 1 \leq j \leq s$ are Borel subsets of $i z(\mathrm{~g})^{*}$. This will follow if we can show that the mappings

$$
\varphi_{\alpha}: i C_{\mathrm{min}, \mathrm{z}}^{*} \rightarrow \mathbb{N}_{0}, \quad \mu \mapsto m_{\alpha}(\mu),
$$

$\alpha \in \Delta_{r}^{+}$, are Borel measurable. As all $\varphi_{\alpha}, \alpha \in \Delta_{r}^{+}$, are lower semicontinuous, the assertion follows.

(iii) This follows from Lemma IV.6.

Corollary IV.8. All $C_{m, j, I}^{G}:=C_{m, j, I} \cap H W\left(G, \Delta^{+}\right), m \in \mathbb{N}, 1 \leq j \leq k, I \subseteq$ $\{r+1, \ldots, n\}$, are Borel subsets of $i t^{*}$. In particular, $H W\left(G, \Delta^{+}\right)$is a Borel subset of $i t^{*}$.

Proof. According to Lemma IV.7(ii), it suffices to show that $H W\left(\Delta^{+}, G\right)$ is a Borel subset of $i t^{*}$.

Let $X_{1}, \ldots, X_{n} \in \mathrm{t}$ be generators of the lattice

$$
\Lambda_{G}:=\left\{X \in \mathrm{t}: \exp _{\widetilde{G}}(X) \in \pi_{1}(G)\right\} .
$$

Then

$$
H W\left(G, \Delta^{+}\right)=\left\{\lambda \in H W(G):\left(\forall X \in \Lambda_{G}\right) e^{\lambda(X)}=1\right\}
$$




$$
=\bigcap_{j=1}^{n} \bigcup_{m \in \mathbb{Z}}\left\{\lambda \in H W\left(\Delta^{+}\right): \lambda\left(X_{j}\right) \in 2 m \pi i\right\}
$$

shows that $H W\left(G, \Delta^{+}\right)$is a Borel subset of $H W\left(\Delta^{+}\right)$. But $H W\left(\Delta^{+}\right)$being a countable union of Borel subsets is Borel (cf. Lemma IV.7(ii)). Thus $H W\left(G, \Delta^{+}\right)$ is a Borel subset of $i t^{*}$, proving the corollary.

\section{The Plancherel Theorem}

Now we are going to prove the admissibility of our parametrization $\gamma$ : $H W\left(G, \Delta^{+}\right) \rightarrow \operatorname{Ext}\left(\mathscr{P}\left(D^{2}\right)_{\imath}\right), \lambda \mapsto K^{\lambda}$ and finally derive the Plancherel Theorem for Biinvariant Hilbert Spaces from it. We begin with a description of the character associated to a unitary highest weight representation.

Lemma IV.9. Let $\lambda \in H W\left(\Delta^{+}\right)$and $\left(\pi_{\lambda}, \mathscr{H}_{\lambda}\right)$ an associated highest weight representation of $\tilde{G}$. Let $\Theta_{\lambda}^{K}$ be the character of $F(\lambda)$ and $m_{\alpha}:=\operatorname{dim}_{\mathbb{C}} g_{\mathbb{C}}^{\alpha}$ for all $\alpha \in \Delta$. Then:

(i) If $\lambda$ is non-singular, then

$$
\Theta_{\lambda}(\operatorname{Exp} X)=\prod_{\alpha \in \Delta_{r}^{+}} \frac{1}{\left(1-e^{-\alpha(X)}\right)^{m \alpha}} \prod_{\alpha \in \Delta \hbar, s} \frac{\Theta_{\lambda}^{K}(\operatorname{Exp}(X))}{1-e^{-\alpha(X)}}
$$

for all $X \in i C_{\max }^{0}$.

(ii) If $\left(\pi_{\lambda}, \mathscr{H}_{\lambda}\right)=\left(\cup_{\lambda z}, \mathscr{F}\left(\mathfrak{m}_{\lambda_{z}}^{+}\right)\right)$is the extended metaplectic representation associated to the parameter $\lambda_{z} \in i C_{\mathrm{min}, \mathrm{z}}$, then

$$
\Theta_{\lambda}(\operatorname{Exp} X)=\prod_{\alpha \in \Delta_{r}^{+}} \frac{e^{\left(\lambda_{z}-\rho_{r}, \lambda_{z}\right)(X)}}{\left(1-e^{-\alpha(X)}\right)^{m \alpha(\lambda z)}}
$$

for all $X \in i C_{\max }^{0}$.

Proof. (i) (cf. the proof of [Ne94, Th. II.9]) As $\lambda$ is non-singular, we have $L(\lambda)=N(\lambda)$ by definition. Thus $L(\lambda)$ is $\mathfrak{C}^{-}$-isomorphic to $U\left(\mathfrak{p}^{-}\right) \otimes F(\lambda)$, where the $\mathbb{C}^{-}$action on $\mathcal{U}\left(\mathfrak{p}^{-}\right) \otimes F(\lambda)$ is given by

$$
X .(Y \otimes v)=[X, Y] \otimes v+Y \otimes X . v
$$

for all $X \in \mathfrak{l}_{\mathbb{C}}, Y \in \mathcal{U}\left(\mathfrak{p}^{-}\right), v \in F(\lambda)$. By the theorem of Poincaré-Birkhoff-Witt,

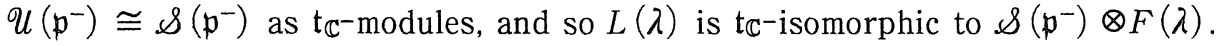
For each $\alpha \in \mathfrak{t}_{\mathbb{C}}^{*}$ we write $\mathbb{C}_{\alpha}$ for the one dimensional $\mathfrak{t}_{\mathbb{C}}$-module of weight $\alpha$. Then $\operatorname{dim}_{\mathbb{C}} g_{\mathbb{C}}^{\alpha}=1$ for all $\alpha \in \Delta_{n, s}$ (cf. [Ne99, Lemma VII.2.3]) implies that

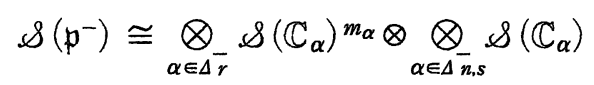

as $\mathfrak{t}_{\mathbb{C}^{-}}$modules. Thus we obtain for all $X \in C_{\max }^{0}$ that 


$$
\begin{aligned}
\Theta_{\lambda}(\operatorname{Exp}(X)) & =\operatorname{tr} \pi_{\lambda}(\operatorname{Exp}(X))=\Theta_{\lambda}^{K}(\operatorname{Exp}(X)) \sum_{\beta \in \Delta_{n, s}^{+}}\left(\sum_{\alpha \in \Delta_{r}^{+}} \prod_{n_{\alpha, n}, n_{\beta} \in \mathbb{N}_{0}} e^{-n_{\alpha} \alpha(X)} e^{-n_{\beta} \beta(X)}\right)^{m_{\alpha}} \\
& =\Theta_{\lambda}^{K}(\operatorname{Exp}(X)) \prod_{\alpha \in \Delta_{r}^{+}} \frac{1}{\left(1-e^{-\alpha(X)}\right)^{m_{\alpha}}} \prod_{\alpha \in \Delta_{n, s}^{+}} \frac{1}{1-e^{-\alpha(X)}}
\end{aligned}
$$

proving the assertion.

(ii) It follows from the discussion in [Ne96, Sect. III] that $L(\lambda)$ is $t_{\mathbb{C}}$-isomorphic to

$$
\mathscr{\&}\left(\bigoplus_{\alpha \in \Delta_{\bar{r}}^{-}}\left(g_{\mathbb{C}}^{\alpha} / W^{\alpha}\left(\lambda_{z}\right)\right)\right) \otimes F\left(\lambda_{z}-\rho_{r, \lambda}\right),
$$

where $F\left(\lambda_{z}-\rho_{r, \lambda}\right)=\mathbb{C}_{\lambda_{z}-\rho r, \lambda}$ is the one dimensional $\mathfrak{t}_{\mathbb{C}}$-module associated to the weight $\lambda_{z}-\rho_{r, \lambda} \in i_{\mathfrak{z}}(\mathfrak{f}) *$. Now the computation of $\left.\Theta_{\lambda} \circ \operatorname{Exp}\right|_{i C_{\max }^{0}}$ is analogous to the one in (i).

Lemma IV.10. For all $m \in \mathbb{N}, 1 \leq j \leq k, I \subseteq\{r+1, \ldots, n\}$ the mapping

$$
\gamma_{m, j, I}: C_{m, j, I}^{G} \rightarrow \operatorname{Hol}(D \times \bar{D}), \lambda \mapsto K^{\lambda}
$$

is continuous.

Proof. First we reduce to the case, where $G$ is simply connected. Let $\widetilde{D}$ $:=\tilde{G} \operatorname{Exp}\left(D_{h}\right)$ be the simply connected covering of $D$ and $\operatorname{Hol}(D \times \bar{D}) \rightarrow \operatorname{Hol}(\widetilde{D} \times$ $\overline{\widetilde{D}})$ the embedding induced from the covering $\widetilde{D} \rightarrow D$. We obtain a commutative diagram

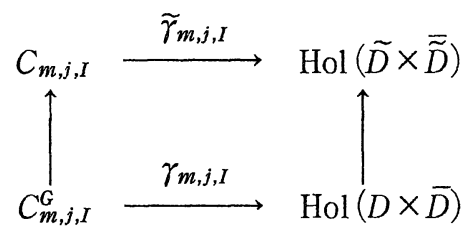

and all vertical arrows in this diagram are embeddings. Thus w.l.o.g. we may assume that $G$ and $D$ are simply connected.

As $K^{\lambda}(s, t)=\Theta_{\lambda}\left(s t^{*}\right)$ for all $s, t \in D$, it suffices to show that the mapping

$$
\sigma_{m, j, I}: C_{m, j, I} \rightarrow \operatorname{Hol}(D),\left.\lambda \mapsto \Theta_{\lambda}\right|_{D}
$$

is continuous.

We write $C(\operatorname{Exp}(\mathscr{D}))^{\mathscr{W}_{\mathfrak{E}}}$ for the Weyl-group invariant continuous functions on $\mathscr{D}$ equipped with the topology of compact convergence. First we show the continuity of the mapping

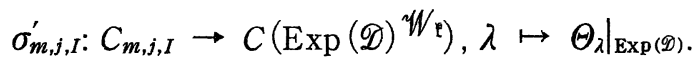


Let $\lambda \in C_{m, j, I}$. By metaplectic factorization we have $\left(\pi_{\lambda}, \mathscr{H}_{\lambda}\right) \cong\left(\nu_{\lambda z} \otimes \pi_{\lambda l}\right.$, $\left.\mathscr{F}\left(\mathrm{m}_{\lambda_{z}}^{+}\right) \widehat{\otimes} \mathscr{H}_{\lambda_{1}}\right)$ and accordingly $\Theta_{\lambda}=\Theta_{\lambda_{z}} \cdot \Theta_{\lambda_{1}}$. For each $1 \leq j \leq n$ we set $\lambda_{j}:=$ $\left.\lambda_{\mathrm{t}}\right|_{i \mathrm{t}_{0}}$ and $\lambda_{0}:=\left.\lambda_{\mathrm{l}}\right|_{i \mathrm{i}_{0}}$. Then

$$
\left(\pi_{\lambda_{1}}, \mathscr{H}_{\lambda_{1}}\right) \cong\left(\pi_{\lambda_{0}}, \mathbb{C}_{\lambda_{0}}\right) \otimes \bigotimes_{j=1}^{n}\left(\pi_{\lambda_{j}}, \mathscr{H}_{\lambda_{j}}\right)
$$

where $\left(\pi_{\lambda_{0}}, \mathbb{C}_{\lambda_{0}}\right)$ is one-dimensional representation of $\tilde{T_{0}}$ with weight $\lambda_{0}$ and $\left(\pi_{\lambda j}, \mathscr{H}_{\lambda_{j}}\right), 1 \leq j \leq n$, is a highest weight representation of $\widetilde{S_{j}}$ with highest weight $\lambda_{j}$. Accordingly we have $\Theta_{\lambda_{1}}=\Theta_{\lambda_{0}} \cdot \prod_{j=1}^{n} \Theta_{\lambda_{j}}$ and so

$$
\Theta_{\lambda}=\Theta_{\lambda_{z}} \cdot \Theta_{\lambda_{0}} \cdot \prod_{j=1}^{n} \Theta_{\lambda_{j}}
$$

Now Lemma IV.7 and Lemma IV.9 imply that there exists an analytic function $f_{m, j, I}$ on $\mathscr{D}$ such that

$$
\Theta_{\lambda}(\operatorname{Exp}(X))=f_{m, j, I}(X) \cdot \Theta_{\lambda}^{K}(\operatorname{Exp}(X))
$$

holds for all $X \in \mathscr{D}, \lambda \in C_{m, j, I}$. By Weyl's Character Formula $\left.\Theta_{\lambda}^{K}\right|_{\operatorname{Exp} \mathscr{D}}$ depends continuously on $\lambda$. Therefore (4.1) implies the continuity of $\sigma_{m, j, I}^{\prime}$.

We write $C\left(\operatorname{Exp}\left(D_{h}\right)\right)^{G}$ for the $\operatorname{Ad}(G)$-invariant continuous function on $\operatorname{Exp}\left(D_{h}\right)$ equipped with the topology of compact convergence. Recall from [Ne99, Th. X III .2.2] that the restriction mapping $C(\operatorname{Exp}(D))^{G} \rightarrow C(\operatorname{Exp}(\mathscr{D}))^{W_{\mathrm{t}}}$ is a continuous bijection, thus an isomorphism by the Open Mapping Theorem. Therefore the continuity of $\sigma_{m, j, I}^{\prime}$ implies the continuity of

$$
\sigma_{m, j, I}^{\prime \prime}: C_{m, j, I} \rightarrow C\left(\operatorname{Exp}\left(D_{h}\right)\right)^{G},\left.\lambda \mapsto \Theta_{\lambda}\right|_{\operatorname{Exp}\left(D_{h}\right)} .
$$

Now we can prove the continuity of $\sigma_{m, j, I}$. Let $\lambda_{n} \rightarrow \lambda$ in $C_{m, j, I}$. We claim that $\left\{\Theta_{\lambda n}: n \in \mathbb{N}\right\}$ is locally bounded. Let $A \subseteq D$ be a compact subset. Then $A$ $\subseteq G \operatorname{Exp}\left(2 A^{\prime}\right)$, where $A^{\prime} \subseteq D_{h}$ is a compact subset. We obtain for all $g \operatorname{Exp}(X)$ $\in A$ that

$$
\begin{aligned}
\left|\Theta_{\lambda}(g \operatorname{Exp}(X))\right| & =\left|\operatorname{tr}\left(\pi_{\lambda}(g \operatorname{Exp}(X))\right)\right|=\left|\operatorname{tr}\left(\pi_{\lambda}\left(g \operatorname{Exp}\left(\frac{1}{2} X\right)\right) \pi_{\lambda}\left(\operatorname{Exp}\left(\frac{1}{2} X\right)\right)\right)\right| \\
& =\left|\left\langle\pi_{\lambda}\left(g \operatorname{Exp}\left(\frac{1}{2} X\right)\right), \pi_{\lambda}\left(\operatorname{Exp}\left(\frac{1}{2} X\right)\right)\right\rangle\right| \\
& \leq\left\|\pi_{\lambda}\left(g \operatorname{Exp}\left(\frac{1}{2} X\right)\right)\right\|_{2} \cdot\left\|\pi_{\lambda}\left(\operatorname{Exp}\left(\frac{1}{2} X\right)\right)\right\|_{2} \\
& \left.\leq\left\|\pi_{\lambda}\left(\operatorname{Exp}\left(\frac{1}{2} X\right)\right)\right\|_{1}^{2}=\left(\Theta_{\lambda}\left(\operatorname{Exp}\left(\frac{1}{2} X\right)\right)\right)^{2} \leq \sup _{z \in \operatorname{Exp}\left(A^{\prime}\right)}\left(\Theta_{\lambda}(z)\right)\right)^{2} .
\end{aligned}
$$

As $\sigma_{m, j, I}^{\prime \prime}$ is continuous, $\left\{\left.\Theta_{\lambda_{n}}\right|_{\operatorname{Exp}\left(D_{h}\right)}: n \in \mathbb{N}\right\}$ is locally bounded, and the claim now follows from (4.2).

Now Montel's Theorem applies and yields a subsequence of $\left(\lambda_{n_{k}}\right)_{k \in \mathbb{N}}$ of 
$\left(\lambda_{n}\right)_{n \in \mathbb{N}}$ such that $\Theta:=\lim _{k \rightarrow \infty} \Theta_{n_{k}}$ exists in Hol $(D)$. As $\sigma_{m, j, I}^{\prime \prime}$ is continuous we must have $\Theta=\Theta_{\lambda}$ on $\operatorname{Exp}\left(D_{h}\right)$, thus $\Theta=\Theta_{\lambda}$, as $\operatorname{Exp}\left(D_{h}\right)$ is a domain of identity in $D$. This proves the continuity of $\sigma_{m, j, I}$ and hence the lemma.

Recall that a topological space $X$ is called Suslin if it is continuous image of a polish space.

\section{Theorem IV.11. The mapping}

$$
\gamma: H W\left(G, \Delta^{+}\right) \rightarrow \operatorname{Ext}\left(\mathscr{P}\left(D^{2}\right)_{i}\right), \lambda \mapsto K^{\lambda}
$$

is an admissible parametrization.

Proof. We know from Proposition IV.4 that $\gamma$ is injective and that im $\gamma$ is a section. We note that $\gamma_{m, j, I}=\left.\gamma\right|_{C_{m, I}^{G}}$ and consider in the following $\gamma_{m, j, I}$ also as a function on $H W\left(G, \Delta^{+}\right)$with support $C_{m, j, I}^{G}$. Then $\gamma=\sum_{m, j, I} \gamma_{m, j, I}$. Thus Lemma IV .10 shows that $\gamma$ is a countable sum of Borel maps, hence Borel.

We claim that $\operatorname{im} \gamma$ is an admissible section. Note that all $C_{m, j, I}^{G}$ are Borel subsets of $i t^{*}$ (cf. Corollary IV.8) and hence Suslin (cf. [Sch73, Ch. II, §1, Th. 3]). Thus by the continuity of $\gamma_{m, j, I}$ the space $\gamma_{m, j, J}\left(C_{m, j, I}^{G}\right)$ is Suslin and

$$
\operatorname{im} \gamma=\bigcup_{m, j, I} \gamma_{m, i, J}\left(C_{m, j, i}^{G}\right)
$$

being a countable union of Suslin spaces is Suslin. In view of [Th94, Th. 1.19], this shows that im $\gamma$ is an admissible section.

Next we show that $\gamma^{-1}$ : $\operatorname{im} \gamma \rightarrow H W\left(G, \Delta^{+}\right)$is universally measurable. We have already seen that $\gamma: H W\left(G, \Delta^{+}\right) \rightarrow \mathrm{im} \gamma$ is an injective Borel map between two Suslin spaces. Thus the assertion follows from von Neumann's Selection Theorem (cf. [Sch73, Ch. II, §3, Th. 13]).

It remains to show that $\left(\gamma^{-1}\right) *(\mathscr{R}(\operatorname{im} \gamma)) \subseteq \mathscr{R}\left(H W\left(G, \Delta^{+}\right)\right)$. Let $\nu$ be a Radon measure on $\operatorname{im} \gamma$ and set $\mu:=\left(\gamma^{-1}\right)^{*}$. $\nu$. We have to show that $\mu$ is a Radon measure on $H W\left(G, \Delta^{+}\right)$.

For all tupels $(m, j, I)$ the prescription $\mu_{m, j, I^{*}}=\left(\gamma_{m, j, I}^{-1}\right) * . \nu$ defines a Radon measure on $H W\left(G, \Delta^{+}\right)$, because $\gamma$ is universally measurable and $\gamma_{m, j, I}$ is continuous (cf. Lemma IV.10). Thus $\nu_{m}:=\sum_{j, I} \nu_{j, m, I}$ being a finite sum of Radon measures is Radon for all $m \in \mathbb{N}$. Recall that there exists a constant $C>0$ such that $\delta_{\min }\left(C_{m}, C_{n}\right)>C$ holds for all $m, n \in \mathbb{N}, m \neq n$, (cf. Lemma IV.7(iii)). As $\nu=$ $\sum_{m \in \mathbb{N}} \nu_{m}$ and $\operatorname{supp}\left(\nu_{m}\right) \subseteq C_{m}, m \in \mathbb{N}$, this shows in particular, that $\nu$ is locally finite and inner regular, hence Radon. This proves the theorem.

Theorem IV.12. (Plancherel Theorem for Biinvariant Hilbert Spaces) Let $D \subseteq \Gamma_{G}\left(W_{\max }\right)$ be a biinvariant domain, $\mathscr{H}_{K} \subseteq \operatorname{Hol}(D)$ be a biinvariant Hilbert 
space associated to $K \in \mathscr{P}\left(D^{2}\right)_{i}$ and $\Delta^{+}$be a positive system associated to $W_{\max }$. For each $\lambda \in H W\left(G, \Delta^{+}\right)$define $K^{\lambda} \in \mathscr{P}\left(D^{2}\right)_{i}$ by $K^{\lambda}(s, t)=\Theta_{\lambda}\left(s t^{*}\right)$ for $s, t \in D$. Then there exists a unique Radon measure $\mu$ on the Borel subset $H W\left(G, \Delta^{+}\right) \subseteq i t^{*}$ such that the following assertions hold:

(i) The kernel $K$ decomposes as

$$
K=\int_{H W\left(G, \Delta^{+}\right)} K^{\lambda} d \mu(\lambda) .
$$

The right hand side is to be understood as an $\mathrm{Hol}(D \times \bar{D})$-valued integral.

(ii) There is a unitary equivalence of $G \times G$ modules

$$
\left(\pi_{K}, \mathscr{H}_{K}\right) \rightarrow\left(\int_{H W\left(G, \Delta^{+}\right)}^{\oplus} \pi_{k}^{c} d \mu(\lambda), \int_{H W\left(G, \Delta^{+}\right)}^{\oplus} B_{2}\left(\mathscr{H}_{\lambda}\right) d \mu(\lambda)\right)
$$

where the measurable structure on the family

$$
\left(B_{2}\left(\mathscr{H}_{\lambda}\right)\right)_{\lambda \in H W\left(G, \Delta^{+}\right)} \cong\left(\mathscr{H}_{K_{\lambda}}\right)_{\lambda \in H W\left(G, \Delta^{+}\right)}
$$

is the one from Proposition II.8.

Proof. We only have to put together Proposition III.7, Proposition IV .3(i) and Theorem IV.11, and the assertions follow.

\section{The Case of Complex Ol'shanskiir Semigroups}

We conclude this section with a discussion of a special case of Theorem IV.12 which is of particular interest, namely where $D=\Gamma_{G}(W)$ is a complex Ol'shanskiı̄ semigroup.

Let $\alpha$ be an absolute value on $\Gamma_{G}(W)$. Then the prescription

$$
\alpha_{0}: i(W \cap \mathrm{t}) \rightarrow \mathbb{R}^{+}, \quad \alpha_{0}(X)=\log \alpha(\operatorname{Exp}(X))
$$

defines a subadditive function, i.e., $\alpha_{0}(X+Y) \leq \alpha_{0}(X)+\alpha_{0}(Y)$ holds for all $X, Y$ $\in i(W \cap \mathrm{t})$.

Lemma IV.13. Let $\left(\pi_{\lambda}, \mathscr{H}_{\lambda}\right)$ be an highest weight representation of $\Gamma_{G} \cdot(W)$ and $\alpha$ an absolute value on $\Gamma_{G}(W)$. Then $\left(\pi_{\lambda}, \mathscr{H}_{\lambda}\right)$ is $\alpha$-bounded if and only if $\lambda \in$ $H W\left(G, \Delta^{+}, \alpha\right)$, where

$$
H W\left(G, \Delta^{+}, \alpha\right):=\left\{\lambda \in H W\left(G, \Delta^{+}\right):(\forall X \in i(W \cap \mathrm{t})) \lambda(X) \leq \alpha_{0}(X)\right\}
$$

Proof. Let $\lambda \in H W\left(G, \Delta^{+}, \alpha\right)$ and note that this is equivalent to

$$
(\forall X \in i(W \cap \mathrm{t})) e^{\lambda(X)}=\left\langle\pi_{\lambda}(\operatorname{Exp}(X)) . v_{\lambda}, v_{\lambda}\right\rangle \leq \alpha(\operatorname{Exp}(X)),
$$

where $v_{\lambda} \in \mathscr{H}_{\lambda}$ is a normalized highest weight vector. Let $\mathrm{t}^{+}:=\{X \in \mathrm{t}:(\forall \alpha \in$ 
$\left.\left.\Delta^{+}\right) i \alpha(X) \geq 0\right\}$. Now we have the inclusion $\mathscr{P}_{L(\lambda)} \subseteq \lambda$-cone $\left(\Delta^{+}\right)$for the weights occuring in $L(\lambda)$ so that $(4.3)$ is equivalent to

(4.4) $\left.\left(\forall X \in i\left(W \cap \mathrm{t}^{+}\right)\right)\left(\forall v \in \mathscr{H}_{\lambda}\right)\right)\left\langle\pi_{\lambda}(\operatorname{Exp}(X)) . v, v\right\rangle \leq \alpha(\operatorname{Exp}(X))\langle v, v\rangle$.

Since all operators $\pi_{\lambda}(\operatorname{Exp}(X)), X \in i W$, are selfadjoint, we have $\left\|\pi_{\lambda}(\operatorname{Exp}(X))\right\|=\sup _{v \in \mathscr{H}_{\lambda} \backslash\{0\}} \frac{\left\langle\pi_{\lambda}(\operatorname{Exp}(X)) \cdot v, v\right\rangle}{\langle v, v\rangle}$.

Now $i(W \cap \mathbf{t}) \rightarrow \mathbb{C}, X \mapsto\left\|\pi_{\lambda}(\operatorname{Exp}(X))\right\|$ is invariant under the big Weyl group $\mathfrak{W}:=N_{K}(\mathrm{t}) / Z_{K}(\mathrm{t})$ so that $\mathbb{W} . \mathrm{t}^{+}=\mathrm{t}$ implies that $(4.4)$ is equivalent to

$$
(\forall X \in i(W \cap \mathrm{t}))\left\|_{\pi_{\lambda}}(\operatorname{Exp}(X))\right\| \leq \alpha(\operatorname{Exp}(X)) .
$$

Note that we can reconstruct $W^{0}$ from $W^{0} \cap \mathbf{t}$, i.e., $W^{0}=\operatorname{Ad}(G) .\left(W^{0} \cap \mathbf{t}\right)$ (cf. [KrNe96, Th. X.3]). This together with the $G$-biinvariance of $\alpha$ shows that $(4.5)$ is equivalent to

$$
\left(\forall s \in \Gamma_{G}(W)\right)\left\|\pi_{\lambda}(s)\right\| \leq \alpha(s),
$$

i.e., $\left(\pi_{\lambda}, \mathscr{H}_{\lambda}\right)$ is $\alpha$-bounded. This proves the lemma.

Theorem IV.14. Let $K \in \mathscr{P}\left(\Gamma_{G}(W)^{2}, \alpha\right)_{i}$ and $\left(\pi_{K}, \mathscr{H}_{K}\right)$ the representation of $G \times G$ on the corresponding Hilbert space $\mathscr{H}_{K}$. Then there exists a unique Radon measure $\lambda$ on the Borel subset $H W\left(G, \Delta^{+}, \alpha\right)$ such that the following assertions hold:

(i) The kernel $K$ can be written as

$$
K=\int_{H W\left(G, \Delta^{+}, \alpha\right)} K^{\lambda} d \mu(\lambda)
$$

with convergence of the right hand side in $\operatorname{Hol}\left(\Gamma_{G}(W) \times \overline{\Gamma_{G}(W)}\right)$.

(ii) There is a unitary equivalence

$$
\left(\pi_{K}, \mathscr{H}_{K}\right) \rightarrow\left(\int_{H W\left(G, \Delta^{+}, \alpha\right)}^{\oplus} \pi_{\lambda}^{c} d \mu(\lambda), \int_{H W\left(G, \Delta^{+}, \alpha\right)}^{\oplus} B_{2}\left(\mathscr{H}_{\lambda}\right) d \mu(\lambda)\right.
$$

of $\overline{\Gamma_{G}(W)} \times \Gamma_{G}(W)$-modules.

Proof. This follows Theorem II.12, Lemma III.6(ii), Proposition IV.3(i), Theorem IV.11 and Lemma IV.13.

\section{References}

[EHW83] Enright, T., Howe, R. and Wallach, N., A classification of unitary highest weight modules, Progr. Malh., 40, Birkhäuser, Basel, 1983.

[Fo96] Folland, G., A Course in Abstract Harmonic Analysis, CRC Press, Boca Raton, 1996.

[Gaa73] Gaal, S. A., Linear Analysis and Representation Theory, Grundlehren 198, Springer, Berlin, Heidelberg, New York, 1973.

[Gr55] Grothendieck, A., Espaces nucléaires, Mem. AMS, 16 (1955). 
[HiNe93] Hilgert, J. and Neeb, K.-H., Lie semigroups and their Applications, Lecture Notes in Math., 1552, Springer, 1993.

[HiÓ192] Hilgert, J. and Ólafsson, G., Analytic extensions of representations, the solvable case, Jap. J. Math., 18 (1992), 213-290.

[HÓØ91] Hilgert, J., Ólafsson, G. and Ørsted, B., Hardy spaces on affine symmetric spaces, $J$. Reine Angew. Math., 415 (1991), 189-218.

[Kr98a] Krötz, B., On Hardy and Bergman spaces on complex Ol'shanskĩ semigroups, Math. Amn., 312 (1998), 13-52.

[Kr98b] - Norm estimates for unitary highest weight representations, submitted.

[Kr98c] - On the dual of complex Ol'shanskii semigroups, submitted.

[Kr98d] - The formal dimension of spherical highest weight representations on a semisimple symmetric space, submitted.

[KrNe96] Krötz, B. and Neeb, K.-H., On hyperbolic cones and mixed symmetric spaces, J. of Lie Theory, 6 ; 1 (1996), 69-146.

[KNÓ97] Krötz, B., Neeb, K.-H. and Ólafsson G., Spherical Representations and Mixed Symmetric Spaces, Represent. Theory, 1 (1997), 424-461.

[KNÓ98] - Spherical Functions on Mixed Symmetric Spaces, submitted.

[Ne94] Neeb, K.-H., Holomorphic representation theory II, Acta Math., 173:1 (1994), 103-133.

[Ne95] - Holomorphic Representations of Ol'shanskir semigroups, in Semigroups in Algebra, Geometry and Analysis, de Gruyter, New York, 1995.

[Ne96] - Coherent states, holomorphic extensions, and highest weight representations, Pac. J. Math., 174 : 2 (1996), 497-542.

[Ne97] _ - On some classes of multiplicity free representations, Manuscripta Math., 92 (1997), 389-407.

[Ne98] - On the complex and convex geometry of Ol'shanskii semigroups, Ann. Inst. Fourier, 48,1(1998), 149-203.

[Ne99] - Holomorphy and Convexity in Lie Theory, Expositions in Mathematics, de Gruyter, in preparation, preprints avaible from the author.

[0182] Ol'shanskii, G. I., Invariant cones in Lie algebras, Lie semigroups, and the holomorphic discrete series, Funct. Anal. Appl., 15 (1982), 275-285.

[0191] - Complex Lie semigroups, Hardy spaces and the Gelfand-Gindikin program, Diff. Geom. Appl., 1 (1991), 235-246.

[0195] - Cauchy-Szegö kernels for Hardy spaces on simple Lie groups, J. of Lie Theory. $\mathbf{5}$ : 2 (1995), 241-274.

[Pe96] Pevsner, M., Espaces de Bergman d'un semi-groupe complexe, C. R. Acad. Sci., Paris, Sér. I, 322 (1996), 635-640.

[Ro63] Rossi, H., On Envelopes of Holomorphy, Comm. Pure Appl. Math., 16 (1963), 9-17.

[Sch73] Schwartz, L., Radon Measures on arbitrary topological spaces and cylindrical measures, Oxford University Press, London, 1973.

[Th78] Thomas, E. G. F., Integral representations of invariant reproducing kernels, Proceedings Bicentennial Congress Wiskunding Genootschap Part II, Amsterdam, 1978.

[Th94] - Integral representations in conuclear cones, Journal of Convex Analysis, 1:2 (1994), 225-258.

[Tr67] Treves, F., Topological vector spaces, distributions, and kernels, Academic Press, New York, 1967. 